\title{
Giardia spp. and the Gut Microbiota: Dangerous Liaisons
}

\author{
Elena Fekete ${ }^{1,2,3 \dagger}$, Thibault Allain ${ }^{1,2,3 \dagger}$, Affan Siddiq ${ }^{1,2,3}$, Olivia Sosnowski ${ }^{1,2,3}$ and \\ Andre G. Buret ${ }^{1,2,3 *}$ \\ ${ }^{1}$ Department of Biological Sciences, University of Calgary, Calgary, AB, Canada, ${ }^{2}$ Inflammation Research Network, University \\ of Calgary, Calgary, AB, Canada, ${ }^{3}$ Host-Parasite Interactions, University of Calgary, Calgary, AB, Canada
}

\section{OPEN ACCESS}

Edited by:

Simone Mario Caccio,

National Institute of Health (ISS), Italy

Reviewed by:

Steven Singer,

Georgetown University, United States

Anton Aebischer,

Robert Koch Institute (RKI), Germany

Cinzia Cantacessi,

University of Cambridge,

United Kingdom

Federica Berrilli,

University of Rome Tor Vergata, Italy

*Correspondence:

Andre G. Buret

aburet@ucalgary.ca

${ }^{\dagger}$ These authors share first authorship

Specialty section:

This article was submitted to

Microbial Symbioses,

a section of the journal

Frontiers in Microbiology

Received: 16 October 2020

Accepted: 15 December 2020

Published: 12 January 2021

Citation:

Fekete E, Allain T, Siddiq A, Sosnowski O and Buret AG (2021) Giardia spp. and the Gut Microbiota:

Dangerous Liaisons.

Front. Microbiol. 11:618106.

doi: 10.3389/fmicb.2020.618106
Alteration of the intestinal microbiome by enteropathogens is commonly associated with gastrointestinal diseases and disorders and has far-reaching consequences for overall health. Significant advances have been made in understanding the role of microbial dysbiosis during intestinal infections, including infection with the protozoan parasite Giardia duodenalis, one of the most prevalent gut protozoa. Altered species composition and diversity, functional changes in the commensal microbiota, and changes to intestinal bacterial biofilm structure have all been demonstrated during the course of Giardia infection and have been implicated in Giardia pathogenesis. Conversely, the gut microbiota has been found to regulate parasite colonization and establishment and plays a critical role in immune modulation during mono and polymicrobial infections. These disruptions to the commensal microbiome may contribute to a number of acute, chronic, and postinfectious clinical manifestations of giardiasis and may account for variations in disease presentation within and between infected populations. This review discusses recent advances in characterizing Giardia-induced bacterial dysbiosis in the gut and the roles of dysbiosis in Giardia pathogenesis.

Keywords: Giardia duodenalis, microbiota, biofilm, polymicrobial infections, probiotics, giardiasis, tissue barrier, pathobiont

\section{INTRODUCTION}

The Protozoan Giardia duodenalis is one of the most common human parasitic enteropathogens worldwide, infecting on average $2 \%$ of adults and $8 \%$ of children in developed countries and up to $33 \%$ of individuals in the developing world (Dunn and Juergens, 2020). Giardia infections are also common in livestock and companion animals and are responsible for significant global health and economic burdens. Infective Giardia cysts are typically ingested via contaminated food or water or via the fecal-oral route. Excystation begins in the stomach, where cyst walls are weakened by host proteases and acidic $\mathrm{pH}$, and is completed in the upper small intestine with the combined action of host and Giardia proteases. Vegetative trophozoites are released into the small intestinal lumen, where they divide by binary fission and attach to the host epithelium via a ventral adhesive disk. The life cycle is completed when trophozoites detach and begin to move further along the GI tract, where they are exposed to bile, and initiate encystation to form new cysts that are excreted into the environment. Acute giardiasis typically causes diarrhea, abdominal pain, nausea, intestinal malabsorption, and weight loss. However, symptoms can occur along a broad spectrum, and asymptomatic infection is common 
(Halliez and Buret, 2013). Giardia may even have protective effects against diarrheal disease in some settings, particularly in children exhibiting polymicrobial infections. In contrast, Giardia infection has also been associated with development of post-infectious complications including irritable bowel syndrome (IBS) and chronic fatigue and has been associated with cognitive defects and stunted growth in children (Halliez and Buret, 2013; Allain and Buret, 2020). The precise mechanisms that govern Giardia pathogenesis are incompletely understood, however, recent research has emphasized important roles for the microbiota.

The intestinal microbiota plays a critical role in homeostasis of the gut and overall health and is frequently found to be altered during gastrointestinal disease. Giardia interacts both directly and indirectly with the microbiome, and through these interactions can modulate host metabolism, immune responses, pain signaling, and the mucus barrier, all of which may have systemic effects that potentially persist even after parasite clearance. Functional and compositional changes to the intestinal microbiota have been demonstrated during the course of Giardia infections, including disruption of the microbial biofilm structure, altered virulence in commensal species, and altered species abundance and diversity. In turn, the disrupted microbiota plays a role in Giardia pathogenesis, influencing colonization resistance, immune responses, and brush border defects. Future research is required to define the role of the microbiota in other aspects of Giardia pathogenesis.

Understanding the role of the intestinal microbiota in Giardia pathogenesis will help to develop therapeutics for a broad range of disorders that implicate disruptions of gut commensal microbes. Recent research has focused on the use of probiotics in the prevention and treatment of giardiasis, as several probiotic microbes have been found to have anti-Giardia effects in vitro and in vivo. Dietary interventions may also contribute to therapy, as the diet has been shown both to alter the microbiome and to influence Giardia pathogenesis. Identification of Giardiaassociated microbiome signatures has also been attempted, which may allow for more targeted studies of the roles of specific commensals during Giardia infection. In this review, we provide state-of-the-art information on the interactions between Giardia and the gut microbiota, and their roles in the pathogenesis of giardiasis.

\section{THE INTESTINAL MICROBIOME}

\section{Structure and Composition of the Intestinal Microbiota}

The intestinal microbiome, encompassing bacteria, viruses, protozoa, and fungi, is a complex and dynamic community of microorganisms that exists in close association with the host intestinal mucosa. Interactions between the gut microbiome and the host have been shown to be important for health and homeostasis. In particular, the bacterial component of the microbiome may play critical roles in the development and persistence of intestinal and extra-intestinal disorders and will be the focus of this review (Carding et al., 2015; Shreiner et al., 2015; Nagao-Kitamoto et al., 2016; Buret et al., 2019).

Dysbiosis, broadly defined as a maladaptive alteration to the bacterial species composition, diversity, or abundance relative to bacterial communities in a healthy individual, has been demonstrated in multiple diseases and disorders of the gastrointestinal tract, including infectious diseases (i.e., bacterial, viral, fungal, and parasitic), irritable bowel syndrome (IBS), inflammatory bowel disease (IBD), and intestinal cancers (Carding et al., 2015; Shreiner et al., 2015; Iacob and Iacob, 2019). The intestinal microbiome plays a major role in the development and maintenance of gut homeostasis, and therefore its disruption may contribute both to initiation and progression of intestinal disease (Wu and $\mathrm{Wu}, 2012$; Lin and Zhang, 2017). Important functions of the microbiome include digestion and absorption of nutrients, immune maturation and modulation, regulation of host cell proliferation and function, modulation of intestinal permeability, transit, and neurotransmission, and defense against opportunistic pathogens via niche exclusion (Lynch and Pedersen, 2016). High microbial diversity is a hallmark of the microbiota's functional redundancy and contributes to its stability, resilience, and adaptability (Tuddenham and Sears, 2015).

Although the composition and abundance of bacterial taxa are highly variable between individuals, it has been suggested that healthy individuals share a "core" microbiota. Recently, the MiBioGen consortium initiative launched a meta-analysis project to better characterize the human microbiome (Wang et al., 2018). In humans, the most prevalent phyla include Firmicutes and Bacteroides, followed by Actinobacteria, Proteobacteria, and Verrucomicrobia (Lozupone et al., 2012; Tuddenham and Sears, 2015). Within these phyla, the specific species composition and their relative abundances can vary dramatically, influenced by genetics, diet, immune status, sex, and age. Microbiota composition also varies throughout an individual's intestinal tract, with dramatic differences between the intestinal mucosa and luminal environment, and longitudinally along different segments of the GI tract (Lozupone et al., 2012; Hollister et al., 2014; Tuddenham and Sears, 2015). Although the adult intestinal microbiota is generally stable, significant shifts may occur due to antibiotic treatment, intestinal infection, or with long-term changes to the diet (Tuddenham and Sears, 2015).

In the small intestine, environmental conditions are generally harsh due to rapid transit times and the periodic influx of digestive enzymes, bile, and stomach contents. As a result, bacterial populations tend to be more dynamic, but less diverse, and with lower overall biomass than in the large intestine (Kastl et al., 2020). Bacterial population density varies along the small intestine, increasing from approximately $10^{4-5}$ $\mathrm{CFU} / \mathrm{ml}$ in the duodenum to $10^{7-8} \mathrm{CFU} / \mathrm{ml}$ in the distal ileum (Kastl et al., 2020). Community composition also varies due to changing physical conditions, such as oxygen and $\mathrm{pH}$ gradients, with the proportion of Gram positive to Gram negative bacteria and the abundance of facultative anaerobic and strict anaerobic species increasing from proximal small intestine to the distal small intestine and colon (Tropini et al., 2017; Kastl et al., 2020). Genera commonly found in the small 
intestine include Lactobacillus, Clostridium, Staphylococcus, Streptococcus, and Bacteroides (Hayashi et al., 2005; Wang et al., 2005; Ahmed et al., 2007). In the colon, the luminal environment tends to be more stable, and as a result, diversity and overall abundance of microbes is higher than in the small intestine, reaching up to $10^{11-12}$ cells/g of luminal contents (Guarner and Malagelada, 2003). Mucinophilic bacteria, such as Akkermansia muciniphila and certain Bacteroides species, have been identified in the outer mucus layers adjacent to the lumen, while closer to the mucosa, oxygen gradients select for more aerotolerant taxa such as Proteobacteria and Actinobacteria (Tropini et al., 2017; Flynn et al., 2018). The proximal colonic mucosa tends to harbor facultative anaerobes including Actinomyces and Enterobacteraceae, and aerobic Pseudomonas. The distal mucosa is more likely to host strict anaerobes including Porphyromonas, Anaerococcus, Finegoldia, and Peptoniphilus (Flynn et al., 2018).

Commensal intestinal microbes form poly-microbial structures known as biofilms, which facilitate adhesion to the mucus gel and help microbes withstand shear forces. Biofilms create complex and unique microenvironments within a self-produced polymeric extracellular matrix and allow for cooperative interactions within and between microbial species. Sessile biofilm bacteria have radically different physiology than planktonic bacteria, creating distinct differences between the bacterial populations found in the intestinal lumen and those associated with the mucosal surface (de Vos, 2015). Biofilms also tend to have increased tolerance against shifting environmental conditions, antibiotics, and the host immune system compared to free-swimming microbes, making them an important structure for maintaining stable commensal populations (Jefferson, 2004; Burmolle et al., 2014; de Vos, 2015). Disruption of biofilm structure and subsequent release of biofilm bacteria may therefore contribute to intestinal disease development and progression by destabilizing commensal populations, and/or by driving functional and phenotypic changes in commensal microbes (Beatty et al., 2017; Buret et al., 2019).

\section{Gut Microbiota Dysbiosis in Protozoan Infections}

Protozoan infections in humans and other mammals have frequently been associated with dysbiosis (Burgess et al., 2017). Common protozoan infections in humans include Giardia duodenalis, Entamoeba histolytica, Cyclospora cayetanensis, Cryptosporidium spp., and Blastocystis spp. (Haque, 2007; Hamad et al., 2016; Iebba et al., 2016). Briefly, protozoan parasites, including Giardia and Cryptosporidium, identified in fecal samples from Columbian children, have been associated with altered microbiome profiles compared to uninfected children (Toro-Londono et al., 2019). Individuals infected with Entamoeba histolytica, the causative agent of amebiasis, were found to have a decreased abundance of protective bacteria generally associated with gut health (Verma et al., 2012). Cryptosporidium was found to alter microbiota composition in mouse models as well as in primates (Coquerel's sifakas - Propithecus coquerel), and dysbiosis was associated with enhanced parasite growth (Ras et al., 2015;
McKenney et al., 2017; Charania et al., 2020). Individuals infected with the protist Blastocystis show increased diversity of the gut microbiota in their fecal contents (Audebert et al., 2016; Nieves-Ramirez et al., 2018). Interestingly, increased microbial diversity is generally associated with a healthier gut (Scanlan et al., 2014; Audebert et al., 2016). Overall, there appears to be a close association between protozoan infection and dysbiosis, with unique dysbiotic profiles associated with individual protozoa. More research is needed to determine whether and how dysbiosis may promote parasitic colonization and contribute to pathogenesis; however, dysbiosis is not exclusively linked to increased disease severity. Rather, some cases of protozoan-induced dysbiosis may lead to positive or neutral effects on gut health.

\section{ROLE OF THE MICROBIOME IN GIARDIA PATHOGENESIS}

\section{The Gut Microbiota Influences Giardia Colonization and Persistence}

The intestinal microbiota plays a key role in determining susceptibility or resistance to colonization by Giardia. Mice from different commercial breeders, possessing distinct microbiomes, were found to have different susceptibilities to Giardia infection (Singer and Nash, 2000). Resistance was found to be transferable to normally susceptible mice via co-housing and was eliminated by antibiotic treatment (Singer and Nash, 2000). Further evidence for this role comes from experimental infection of germ-free mice, which show higher fecal cyst counts than conventionally raised mice likely due to both the immaturity of the immune system and the absence of niche competition by commensal microbes (Torres et al., 1992). It is worth mentioning that antibiotic treatment is often required for robust infection of mice with human Giardia isolates, suggesting that the microbiome may play a role in establishment and persistence of infection. However, it is important to consider that antibiotic treatment itself may induce dysbiosis. Challenges arise in the study of immune responses, mucosal disruption, bacterial translocation, and pain responses when antibiotics are used, as antibiotic-induced dysbiosis can itself cause disruptions in these areas. Despite these challenges, antibiotics remain a useful tool to study many human isolates of Giardia in vivo.

Susceptibility to Giardia infection varies significantly among different age groups in humans and in animal models, which is likely due at least in part to age-related shifts in microbiota composition, as well as changes in host immune factors. Several studies have shown the efficacy of using a suckling mouse model to study infection with Giardia isolates that do not typically infect adult mice (Hill et al., 1983; Allain et al., 2017, 2018a; Riba et al., 2020). Shifts in the microbiota that occur upon weaning may be responsible for this increased resistance to Giardia infection (Hill et al., 1983). Similarly, in human populations, children aged 6 months to 5 years show higher susceptibility to Giardia infection than other age groups (Lengerich et al., 1994; Harvey et al., 2020). The microbiota 
of human infants is distinctly different from that of adults and is continuously evolving through the first several years of life (Lynch and Pedersen, 2016). In the first 6 months of life, maternal milk confers passive immunity. In addition, both via direct microbe-trophozoite interactions and by influencing cell signaling pathways and development of the immune system, the microbiota appears to be a critical determinant of susceptibility or resistance to Giardia infection.

\section{Microbiota-Immune System Interactions During Giardia Infection}

Giardia duodenalis is a non-invasive enteropathogen that does not trigger an overt inflammatory response. However, both innate and adaptive immunity are required for control and clearance of Giardia. Critical roles have been identified for the cytokines IL-6 and IL-17, as well as for secretory IgA (Langford et al., 2002; Zhou et al., 2003; Dreesen et al., 2014; Dann et al., 2015). CD4+ and CD8+ T cells play important roles in parasite clearance and pathogenesis, respectively (Scott et al., 2004; Keselman et al., 2016). In addition, mucosal mast cells and macrophages appear to play a role in parasite clearance, while nitric oxide and antimicrobial peptides produced by intestinal cells may have direct or indirect cytostatic effects against Giardia trophozoites (Belosevic and Daniels, 1992; Aley et al., 1994; Li et al., 2004; Stadelmann et al., 2013; Fink et al., 2019). However, recent findings have demonstrated that Giardia is capable of modulating host immunity. Giardia may directly cleave host-produced chemokines and cytokines, resulting in dampened immune responses (Cotton et al., 2014a,b; Liu et al., 2018, 2019; Ortega-Pierres et al., 2018; Allain et al., 2019). Furthermore, Giardia may avoid host adaptive immunity through the expression of variant-specific surface proteins (VSPs; Langford et al., 2002; Hjollo et al., 2018).

Immune modulation by the microbiota may play a key role in several aspects of Giardia pathogenesis. Giardia infection is associated with diffuse shortening of the brush border microvilli and disaccharidase deficiencies, which leads to malabsorption and maldigestion of nutrients (Buret et al., 1992; Scott et al., 2004). These effects are driven by CD8+ T cells and transfer of $\mathrm{CD} 8+\mathrm{T}$ cells from infected mice to naïve animals is able on its own to drive brush border injury and malfunction (Scott et al., 2004; Keselman et al., 2016). Antibiotic treatment was found to attenuate these Giardia-induced histopathological alterations and disaccharidase deficiencies by preventing the activation of $\mathrm{CD} 8+\mathrm{T}$ cells. Interestingly, antibiotic treatment did not affect $\mathrm{CD} 4+\mathrm{T}$ cell activation. CD4+ $\mathrm{T}$ cells play an important role in parasite clearance, and so the reductions in intestinal pathologies in these mice were not due to reduced trophozoite burden (Scott et al., 2004; Keselman et al., 2016). Similarly, histopathological alterations were found to be less severe in germ-free mice than conventional mice infected with Giardia. This may be similarly attributed to defects in the host immune response, as germ-free mice are considered to have "immature" immune systems. In comparison to germ-free mice, Giardia-infected conventional mice showed higher levels of IgA in the intestinal fluid and higher levels of IgG and IgM in the serum, further corroborating that the microbiota plays important roles in the adaptive immune response during Giardia infection and is a driving factor in some aspects of Giardia pathogenesis (Torres et al., 2000).

The microbiota can also influence the expression of cytokines during Giardia infection. In germ-free mice reconstituted with human colonic microbial communities, mice that received microbes previously exposed to G. duodenalis trophozoites (isolate NF) showed increased production of proinflammatory cytokines, as well as proliferation and enlargement of lymphocyte aggregations within the follicles, compared to mice receiving control bacteria. Mice receiving Giardiamodified microbial communities showed increased production of IL- 6 , IL- $1 \beta$, TNF- $\alpha$, and IFN- $\gamma$, suggesting Giardia may alter microbial communities in such a way as to promote a pro-inflammatory response that is driven by the microbes themselves (Beatty et al., 2017).

While the microbiome may modulate immune responses to Giardia, Giardia in turn may initiate and alter immune responses in ways that contribute to dysbiosis, either through direct effects on microbes or by altering the intestinal microenvironment. Giardia has been found to induce Th17 type immune responses, with elevated levels of IL-17 being observed both in mouse models of infection and in Brazilian children infected with Giardia (Dreesen et al., 2014; Dann et al., 2015; Cascais-Figueiredo et al., 2019; Singer et al., 2019). IL-17A is an important mediator of anti-microbial responses and plays a role in the transport of IgA across the epithelium and into the intestinal lumen, where it creates an anti-microbial chemical barrier to protect the underlying epithelium (Dann et al., 2015; Singer et al., 2019). IgA also plays a role in Giardia clearance, targeting Giardia VSPs (Langford et al., 2002; Hjollo et al., 2018). Increased levels of IgA were similarly seen in the saliva and serum of Giardia-infected Egyptian children and in mice treated with Giardia excretory/secretory products (ESPs; El-Gebaly et al., 2012; Jimenez et al., 2014; Hjollo et al., 2018). In IL-6 deficient mice, Th17 cell development is impaired, whereas IgA production is unaffected, and the clearance of Giardia infection is delayed (Bienz et al., 2003; Dann et al., 2015). Increased eosinophil numbers have been observed during Giardia infection, and in eosinophil deficient mice, IL-17 levels, as well as serum and luminal IgA levels, are decreased suggesting elevated levels of IL-17 and IgA during Giardia infection are dependent in part on eosinophil activity (Jung et al., 2015).

Specific members of the commensal microbiota have been implicated in driving immune responses during Giardia infection. Segmented filamentous bacteria (SFB) have been shown to drive IL-17 production (Ivanov et al., 2009; Barash et al., 2017). Mouse colonies that were previously shown to be resistant to Giardia infection (Singer and Nash, 2000) were found to have SFB (quantitative PCR on 16S rRNA), while susceptible mice did not, suggesting SFB may play a role in the protective effects of the microbiota against Giardia colonization. Following oral inoculation of SFB in germ-free mice, isolated T cells and APC cells from the lamina propria were found to have increased IL-17 expression, while fecal IgA was elevated (Atarashi et al., 2015). These observations are in support of the hypothesis that SFB may contribute at least in part to the characteristic 
adaptive immune response observed during Giardia infection. Interestingly, neonatal mice infected with Giardia muris fail to develop a protective IL-17A response and show delayed parasite clearance compared to adult mice (Paerewijck et al., 2019). IL-17A responses and IgA production were only evident after weaning (Paerewijck et al., 2019). Colonization of the GI tract by SFB occurs in rat and mouse hosts at the onset of the weaning process, and thus SFB populations are low in neonates, which may contribute to their inability to mount IL-17 responses and their poor resistance to Giardia infection (Koopman et al., 1987; Ericsson et al., 2014). Similarly, Clostridia have been implicated in promotion of regulatory $\mathrm{T}$ cell responses (Garland et al., 1982; Keselman et al., 2016; Fink and Singer, 2017). Significant reductions in Clostridia have been observed in Giardia-infected mice, which may explain the lack of a significant Treg response typically observed during Giardia infection (Barash et al., 2017). However, this appears to be dependent on mouse strain and genetic background. BALB/c mice infected with Giardia muris showed a more significant Treg response than C57BL/6 mice and shed more Giardia cysts. Interestingly, $\mathrm{BALB} / \mathrm{c}$ mice showed larger populations of Clostridia than the $\mathrm{C} 57 \mathrm{BL} / 6$ mice, further suggesting that presence of certain commensal species may drive specific immune responses (Yordanova et al., 2019). However, in contrast to previous studies, although $\mathrm{BALB} / \mathrm{c}$ mice showed increased SFB populations, they showed reduced Th17 responses (Yordanova et al., 2019). Complex interactions involving multiple species likely drive microbiome-immune system interactions.

To subvert host immune responses, Giardia possesses several mechanisms of immunomodulation. Nitric oxide (NO) is an important antimicrobial compound produced by epithelial cells in the gut. It has broad, non-specific effects against luminal microbes, including Giardia. NO is synthesized from L-arginine, which is a primary energy source for Giardia trophozoites. Due to Giardia's consumption of L-arginine, NO levels are depleted in the gut during infection, resulting in impaired antimicrobial activity at the mucosal surface (Stadelmann et al., 2012; Allain et al., 2017; Fink and Singer, 2017; Singer et al., 2019). Similarly, Giardia can impair host immune response by, at least in part, cleaving cytokines and chemokines such as CXCL1, CXCL2, CXCL3, IL-8, CCL2, and CCL20 in a cysteine protease dependent manner (Cotton et al., 2014a,b; Liu et al., 2018, 2019; Ortega-Pierres et al., 2018; Allain et al., 2019).

The immune system is highly dependent on the microbiota for development and maturation. Studies of immune responses in germ-free mice have revealed that the absence of microbes impacts both innate and adaptive immunity, and thus germfree mice are considered to have "immature" immune systems. For such studies, the use of antibiotic treatment is a common alternative to germ-free animals, as it allows for the study of animals with a depleted gut microbiota, but fully developed immune system. However, as stated earlier, antibiotic treatment will itself alter immune function by causing dysbiosis, and the types and combinations of antibiotics used must be carefully considered, as each may selectively deplete certain microbial populations, as well as modify the host immune response.
Broadly, both antibiotic treated and germ-free animals typically show reduced immune cell populations, altered cytokine production, and disrupted cell signaling pathways. It is important to consider these alterations when studying Giardia using antibiotic treated or germ-free models.

\section{Intestinal Mucus Disruption During Giardiasis: A Role for Microbiota?}

The microbiome and the intestinal mucus layers exist in close association. Mucus acts as a physical and chemical barrier that can prevent inflammatory responses to microbes and other potentially harmful luminal contents (Cornick et al., 2015). It also creates a niche environment for commensal species, providing binding sites and acting as a nutrient source when degraded by mucolytic bacteria such as Bacteroides thetaiotaomicron, Akkermansia sp., or Ruminococcus sp., and therefore facilitates microbial colonization of the gut (Schroeder, 2019). Changes to the intestinal mucus gel have frequently been associated with dysbiosis, potentially as a result of altered nutrient or binding site availability, or due to disrupted barrier function, facilitating the translocation of commensal bacteria through the intestinal epithelium and leading to mucosal immune activation (Schroeder, 2019). In turn, dysbiosis may lead to significant changes to the mucus barrier, as the commensal microbiome plays key roles in regulating production and secretion of mucus by intestinal goblet cells and in goblet cell differentiation (Cornick et al., 2015). Indeed, mucus in the large and small intestines of germ-free mice has altered penetrability and composition compared to conventional mice, which can be normalized by microbiota transfer (Johansson et al., 2015). Further, in mouse colonies that vary in microbiota composition, differences in the penetrability of the inner colonic mucus layer to bacteria-sized beads were observed, suggesting variations in commensal populations, and potentially dysbiosis, may predispose individuals to having a more penetrable mucus barrier and therefore increase the risk of pathogen translocation (Jakobsson et al., 2015). Mice with a more penetrable colonic mucus layer showed increased levels of Proteobacteria compared to those with impenetrable mucus (Jakobsson et al., 2015). Interestingly, Giardia has been shown to drive expansion of Proteobacteria in mouse models of infection (Barash et al., 2017). In addition, various microbes and microbial products have been found to either inhibit or stimulate mucus production and secretion by goblet cells or to change the chemical composition of mucins, thus altering the intestinal microenvironment and potentially barrier function (Cornick et al., 2015; Schroeder, 2019).

Giardia is able to disrupt intestinal mucus (Amat et al., 2017). In mice infected with G. duodenalis, significant thinning of the colonic mucus layer can be observed. In vitro G. duodenalis can degrade purified human MUC2 mucin in a cysteine protease dependent manner. Together, these findings indicate that, during infection, Giardia may cause localized depolymerization of the mucin gel matrix, potentially facilitating the translocation of both the parasite and commensal bacteria. This is consistent with the findings that giardiasis promotes the invasion of commensal bacteria in vivo (Halliez et al., 2016; 
Allain et al., 2017; Beatty et al., 2017; Manko et al., 2017). In addition, $G$. duodenalis strain GS/M alters the expression of both Muc2 and Muc5ac, the predominant secretory mucins expressed in the intestines. Specifically, in vivo, Muc2 and Muc5ac expressions were increased in the colon; while in the proximal jejunum, Muc2 was upregulated and Muc5ac downregulated (Amat et al., 2017). In vitro alterations to mucin gene expression were found to be Giardia cysteine protease dependent (Amat et al., 2017; Fekete et al., 2019). The presence of a functional mucus layer also protects against parasitic colonization, as in MUC2 deficient mice, which lack a functional mucus gel barrier in the small and large intestines, Giardia burden is significantly elevated compared to wild-type mice (Amat et al., 2017).

Although the cause-to-effect relationship between dysbiosis and mucosal disruptions in the context of Giardia infections still requires further investigation, reciprocal relationships likely exist, wherein the disruption of mucus drives bacterial dysbiosis and vice versa. Giardia-driven dysbiosis may result in changes to mucus properties, including glycosylation patterns and penetrability. Changes to populations of mucolytic bacteria may result in altered mucus degradation throughout the gut, even in regions of the gut, like the large intestine, where Giardia does not colonize but still disrupts the mucus layer (Amat et al., 2017). Indeed, an increase in Akkermansia has been demonstrated in G. duodenalis GS/M infected mice (Amat et al., 2017). Therefore, we can hypothesize that altered production and secretion of mucus throughout the gut may also be driven by bacteria or bacterial metabolites. Individual variations in mucus structure and composition as a result of individual microbiome variability may impact susceptibility to Giardia colonization and the severity of symptoms. These effects may persist long after parasite clearance, potentially contributing, at least in part, to development of post-infectious disorders in humans.

\section{Probiotics: The Giardia Kryptonite}

Probiotics, which are defined as "live microorganisms that, when administered in adequate amounts, confer a health benefit to the host," represent a large class of microorganisms, including bacteria and yeast, that may have protective effects against parasitic infection or other GI diseases (Hill et al., 2014). Probiotic strains must meet international food safety agencies criteria for consumption, such as "Generally Recognized as Safe" status (Food and Drug administration criteria) or "Qualified Presumption of Safety" status (European Food Safety Authority; Sorokulova, 2008; Alazzaz, 2020). The largest bacterial group of probiotic strain candidates is lactic acid bacteria, mainly represented by the genus Lactobacillus, which are characterized by the production of lactic acid as an end product of glucose metabolism. Other common and well-characterized probiotic strains belong to other genera such as Bifidobacterium, Enterococcus, and Saccharomyces (yeast). Following oral administration, probiotic microbes must survive gastric acids and bile acid toxicity, compete with the gut commensal microbiota for ecological niches, and adhere to the mucosa in the small intestine (Figueroa-Gonzalez et al., 2011; Markowiak and Slizewska, 2017). In most cases, probiotic strains are screened for pathogen inhibition, either via secretion of bacteriocins, competitive advantage for nutrients, or by stimulating the host immune response against pathogens (Figueroa-Gonzalez et al., 2011; Markowiak and Slizewska, 2017).

Over the past 20 years, numerous studies have explored the anti-Giardia properties of probiotic strains as an alternative strategy for prevention and treatment of giardiasis. In particular, lactobacilli are a good candidate since they share the same ecological niches as Giardia trophozoites in the upper small intestine. In vitro and in vivo studies showed that several lactobacilli species can exert cytostatic and/or cytotoxic effects against Giardia trophozoites (Table 1). Other probiotic strains from other genus or species showed potent anti-Giardia properties ex-vivo and in vivo, such as Bifidobacterium spp., Weissella paramesenteroides, Enterococcus faecium, Saccharomyces boulardii, as well as multi-strain probiotic and mixed bacteria and yeast cultures such as kefir grains (composed of Lactobacillus spp., Lactococcus lactis Leuconostoc mesenteroides, Saccharomyces spp., and Acetobacter spp.) and Slab51 (Lactobacillus spp., Streptococcus thermophilus, and Bifidobacterium spp.; Table 1).

The mechanisms underlying these inhibitory effects remain unclear. Probiotic strains may act like a "Swiss Army Knife" against enteropathogens by secreting anti-microbial factors, competing for ecological niches, enhancing the host innate and adaptive immune response, regulating intestinal barrier integrity, and promoting mucosal healing (Taverniti and Guglielmetti, 2011; Travers et al., 2011). In vivo models of giardiasis revealed that the probiotic supplementation was associated with potent immuno-modulatory effects, with upregulation of anti-inflammatory cytokines, such as IL-10, or increases in Giardia-specific IgA and IgG (Benyacoub et al., 2005; Shukla and Sidhu, 2011). The ability of probiotics to compete for ecological niches is another important aspect of their anti-Giardia effects, as Giardia must resist the hostile microenvironmental conditions of the upper small intestine, where bile and digestive enzymes pour in. For instance, lactobacilli's bile salt hydrolase (BSH) enzymatic activity, a key bacterial cellular process for bile detoxification, is associated with the release and accumulation of deconjugated bile salts, which are toxic for Giardia trophozoites (Travers et al., 2016). Indeed, a recent report showed that the BSH activity of lactobacilli strains is positively correlated with their antiGiardia effects (Allain et al., 2018a). Further investigations showed that recombinant BSHs from the probiotic strain $L$. johnsonii La1 can directly inhibit the growth of Giardia trophozoites in vitro and in vivo (Allain et al., 2017). Other reports suggest that lactobacilli, such as L. acidophilus P106, secrete bacteriocins that exert direct cytotoxic effects on Giardia trophozoites (Amer et al., 2014). Consumption of key metabolites, such as L-arginine, nucleosides, purines, and pyrimidines, by probiotic strains can also create a competitive advantage over trophozoites in the small intestine (Lindmark and Jarroll, 1982; Jarroll et al., 1989; Baum et al., 1993; Jarroll, 2011; Allain et al., 2018b). Finally, other probiotic properties must be considered, such as the presence of adhesion factors and competition for binding sites on enterocytes via carbohydrate-binding specificities (Pique et al., 2019). 
TABLE 1 | Probiotic strains exerting anti-Giardia activities in vitro, ex vivo, and in vivo.

\begin{tabular}{|c|c|c|c|c|c|}
\hline & Giardia isolate & $\begin{array}{l}\text { Anti-Giardia } \\
\text { activity in } \\
\text { vitro }\end{array}$ & $\begin{array}{l}\text { Anti-Giardia activity in } \\
\text { vivo (reduced } \\
\text { parasitic load) }\end{array}$ & Animal model & References \\
\hline Bifidobacterium longum 5(1A) & $\begin{array}{l}\text { Giardia duodenalis strain } \\
\text { GS/M clone } \mathrm{H} 7\end{array}$ & N/A & Yes & $\begin{array}{l}\text { 4-6 weeks Mongolian } \\
\text { gerbils }\end{array}$ & Fonseca et al., 2019 \\
\hline Enterococcus faecium SF68 & $\begin{array}{l}\text { Giardia duodenalis strain } \\
\text { GS/M clone } \mathrm{H} 7\end{array}$ & $\mathrm{~N} / \mathrm{A}$ & Yes & 6 weeks C57BL/6 mice & $\begin{array}{l}\text { Benyacoub et al., } \\
2005\end{array}$ \\
\hline Enterococcus faecium SF68 & Unknown & $\mathrm{N} / \mathrm{A}$ & $\begin{array}{l}\text { Yes (combined with } \\
\text { metronidazole treatment) }\end{array}$ & 16 weeks to 3 years dogs & Fenimore et al., 2017 \\
\hline Lactobacillus brevis CNRZ 1845 & Giardia duodenalis strain WB & Yes & $\mathrm{N} / \mathrm{A}$ & $\mathrm{N} / \mathrm{A}$ & Allain et al., 2018a \\
\hline Lactobacillus casei MTCC 1423 & $\begin{array}{l}\text { Giardia duodenalis strain } \\
\text { Portland-I }\end{array}$ & N/A & Yes & 5-6 weeks BALB/c mice & $\begin{array}{l}\text { Shukla et al., 2008; } \\
\text { Shukla and Sidhu, } \\
2011 \text {; Shukla et al., } \\
2013\end{array}$ \\
\hline Lactobacillus casei ATCC 393 & Giardia duodenalis strain WB & Yes & N/A & $N / A$ & Allain et al., $2018 a$ \\
\hline Lactobacillus crispatus CIP 103606 & Giardia duodenalis strain WB & Yes & $\mathrm{N} / \mathrm{A}$ & $\mathrm{N} / \mathrm{A}$ & Allain et al., 2018a \\
\hline Lactobacillus gasseri CNCM I-4884 & Giardia duodenalis strain WB & Yes & Yes & OF-1 Suckling mice & Allain et al., $2018 a$ \\
\hline Lactobacillus gasseri ATCC 3323 & Giardia duodenalis strain WB & Yes & $\mathrm{N} / \mathrm{A}$ & $\mathrm{N} / \mathrm{A}$ & Allain et al., 2018a \\
\hline Lactobacillus helveticus ATCC 11977 & Giardia duodenalis strain WB & Yes & N/A & $\mathrm{N} / \mathrm{A}$ & Allain et al., $2018 a$ \\
\hline Lactobacillus johnsonii La1,(NCC533) & Giardia duodenalis strain WB & Yes & Yes & $\begin{array}{l}\text { Mongolian gerbils (young } \\
\text { adults) }\end{array}$ & $\begin{array}{l}\text { Perez et al., 2001; } \\
\text { Humen et al.,2005 }\end{array}$ \\
\hline Lactobacillus johnsonii La1,(NCC533) & Giardia duodenalis strain WB & Yes & No & OF-1 Suckling mice & Allain et al., $2018 a$ \\
\hline $\begin{array}{l}\text { Lactobacillus johnsonii CIP, } \\
\text { Lactobacillus johnsonii 103653, } \\
\text { Lactobacillus johnsonii CIP 103614, } \\
\text { Lactobacillus johnsonii CIP } 103654 \text {, } \\
\text { Lactobacillus johnsonii CIP } 103781 \text {, } \\
\text { Lactobacillus johnsonii CIP } 103652 \text {, } \\
\text { Lactobacillus johnsonii CIP } 103786 \text {, } \\
\text { Lactobacillus johnsonii ATCC } 33200 \text {, } \\
\text { Lactobacillus johnsonii CNRZ 218, and } \\
\text { Lactobacillus johnsonii CNRZ 1897 }\end{array}$ & Giardia duodenalis strain WB & Yes & $\mathrm{N} / \mathrm{A}$ & $\mathrm{N} / \mathrm{A}$ & Allain et al., $2018 a$ \\
\hline Lactobacillus reuteri CNRZ 431 & Giardia duodenalis strain WB & Yes & $\mathrm{N} / \mathrm{A}$ & $\mathrm{N} / \mathrm{A}$ & Allain et al., 2018a \\
\hline Lactobacillus rhamnosus GG & $\begin{array}{l}\text { Giardia duodenalis strain } \\
\text { Portland-I }\end{array}$ & N/A & Yes & 5-6 weeks BALB/c mice & $\begin{array}{l}\text { Goyal et al., 2011, } \\
\text { 2013; Goyal and } \\
\text { Shukla, } 2013\end{array}$ \\
\hline Saccharomyces boulardii & Unknown & N/A & $\begin{array}{l}\text { Yes (combined with } \\
\text { metronidazole treatment) }\end{array}$ & Humans (clinical study) & $\begin{array}{l}\text { Besirbellioglu et al., } \\
2006\end{array}$ \\
\hline Saccharomyces boulardii & $\begin{array}{l}\text { Giardia duodenalis strain } \\
\text { BHRA-93 }\end{array}$ & N/A & Yes & $\begin{array}{l}\text { 4-6 weeks Mongolian } \\
\text { gerbils }\end{array}$ & Ribeiro et al., 2018 \\
\hline Weissella paramesenteroides WpK4 & $\begin{array}{l}\text { Giardia duodenalis strain } \\
\text { GS/M clone } \mathrm{H} 7\end{array}$ & $\mathrm{~N} / \mathrm{A}$ & Yes & $\begin{array}{l}\text { 4-6 weeks Mongolian } \\
\text { gerbils }\end{array}$ & Fonseca et al., 2019 \\
\hline kefir grains CIDCA AGK1 ${ }^{1}$ & $\begin{array}{l}\text { Giardia duodenalis strain } \\
\text { GS/M clone } \mathrm{H} 7\end{array}$ & $N / A$ & Yes & 4-5 weeks C57BL/6 mice & Franco et al., 2013 \\
\hline Slab5 $51^{2}$ & Giardia duodenalis strain WB & $\mathrm{N} / \mathrm{A}$ & $\begin{array}{l}\text { Yes (ex vivo anti-Giardia } \\
\text { activity) }\end{array}$ & $\begin{array}{l}\text { Duodenum sections of } \\
\text { CD-1(ICR)BR mice }\end{array}$ & Perrucci et al., 2019 \\
\hline
\end{tabular}

${ }^{1}$ CIDCA AGK1: Lactobacillus plantarum, Lactobacillus kefir, Lactococcus lactis subsp. lactis, Leuconostoc mesenteroides, Saccharomyces spp., and Acetobacter spp. 2Slab51: Streptococcus thermophilus DSM 32245, Bifidobacterium lactis DSM 32246, Bifidobacterium lactis DSM 32247, Lactobacillus acidophilus DSM 32241, Lactobacillus helveticus DSM 32242, Lactobacillus paracasei DSM 32243, Lactobacillus plantarum DSM 32244, and Lactobacillus brevis DSM 27961.

More clinical studies are needed to establish the benefits of probiotics in giardiasis, as well as in other disorders of the gut.

\section{ALTERATIONS TO THE MICROBIOTA DURING GIARDIA INFECTION}

\section{Alterations to the Microbiome in Clinical Studies of Giardiasis}

Several recent studies have begun to shed light on bacterial taxonomic shifts associated with Giardia infections in humans (Figure 1). In a study of individuals from sub-Saharan Africa, microbial taxonomic differences were assessed in males and females ranging from 1 to 74 years of age infected with Giardia and other protozoa. Temporal temperature gradient gel electrophoresis (TTGE) analysis revealed that individuals infected by Giardia alone have a distinct TTGE profile compared with uninfected individuals or those infected with Entamoeba or Blastocystis (Iebba et al., 2016). Dispersion matrices showed that Giardia infection was positively correlated with an increase in Escherichia coli, Enterococcus species, and bifidobacteria. The authors failed to establish a correlation between Giardia infection and a dysbiosis index, which characterized by the ratio between the relative abundances of Faecalibacterium prausnitzii and 
E. coli (Iebba et al., 2016). An early study of patients with Giardia predicted that the small intestinal microbiota composition can influence the pathogenesis and clinical manifestations of giardiasis. Increased jejunal concentrations of enterobacteria (i.e., live bacteria isolated from mucosa and luminal fluids) such as Klebsiella pneumoniae, Enterobacter cloacae, and Enterobacter hafniae were observed only in patients with severe intestinal malabsorption, while enterobacteria overgrowth was not observed in patients with mild malabsorption (Tomkins et al., 1978). In an attempt to investigate the role of microbiotaGiardia interactions in the pathogenesis of giardiasis, 31 facultative and strictly anaerobic duodenal bacteria isolated from patients with symptomatic giardiasis were administered in different combinations to germ-free mice. While bacteria cocktails alone failed to mimic Giardia pathogenesis, co-administration with $G$. duodenalis isolate BT6 resulted in an increase in Giardia-specific IgA, IgM, and IgG levels, suggesting that the small intestine microbiota can enhance the host immune response to Giardia infection (Torres et al., 2000).

$16 \mathrm{~S}$ rRNA gene sequencing analysis of fecal microbiota from Colombian children infected with one or several parasites revealed that individuals infected with Giardia only $(n=5)$ had distinct microbial signatures compared to children with no detectable gut parasites. The Giardia group had a lower Shannon's alpha diversity index and lower species richness compared to uninfected controls (Toro-Londono et al., 2019). Despite recent doubts about the robustness of "enterotypes," it has been suggested that gut bacterial species may be clustered into distinct categories: (1) enterotype I, dominated by Bacteroides; (2) enterotype II, dominated by Prevotella; and (3) enterotype III, dominated by Ruminococcus. These may be influenced by dietary habits and environment (Rinninella et al., 2019). In this study, children infected with Giardia present a shift from enterotype I to enterotype II, characterized by an increase of Prevotella and a decrease of Bacteroides (Toro-Londono et al., 2019). Significant increases in Prevotella were also observed in Argentinian children infected with Giardia compared to uninfected individuals (Mejia et al., 2020). Interestingly, Giardia infection was associated with a decreased abundance of microbiome Vitamin B12 (cobalamin) biosynthesis genes (CbiM), which has been associated with impaired growth and development in children (Mejia et al., 2020).

Recently, a study employed a database mining approach to examine data from the Global Enteric Multicenter Study (GEMS) and "Malnutrition and Enteric Disease Study" (MAL-ED). Human cohorts from four countries were studied to determine whether Giardia infection was associated with a distinct microbiota signature and/or microbiota dysbiosis in young individuals. Among the GEMS participants, 215 individuals

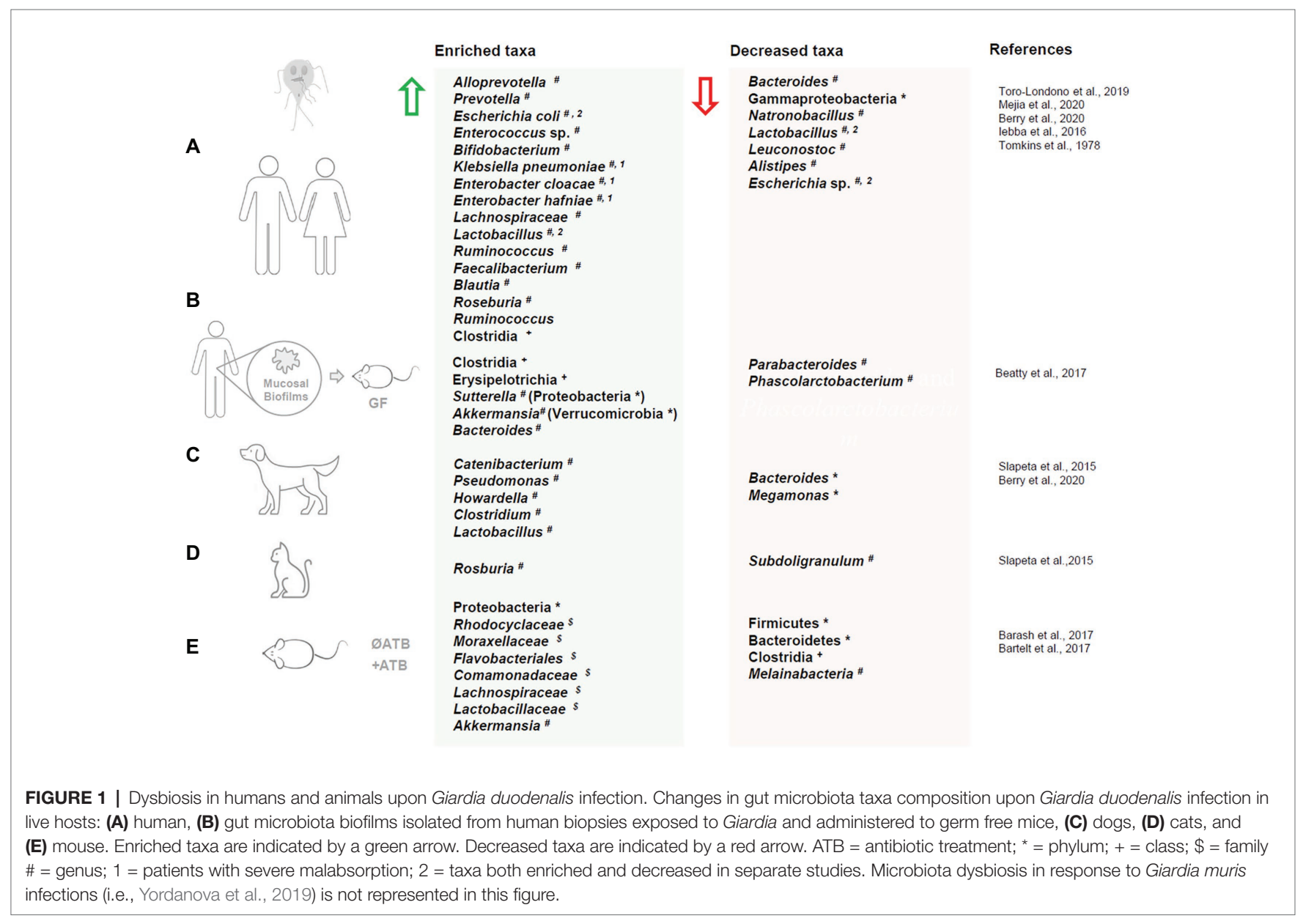


tested positive for Giardia and were subclassified as having either moderate to severe diarrhea (MSD) or no MSD. Metagenomic biomarker discovery analysis (LEfSe score) revealed that Giardia infection was associated with an enrichment of Prevotella and a reduction in Gammaproteobacteria, regardless of the presence of mild to severe diarrhea (Berry et al., 2020). To a lesser extent, Giardia infection was also associated with an increase of Lachnospiraceae, Ruminococcus, and Clostridiales. Conversely, Giardia infection was correlated with a decrease of Natronobacillus, Lactobacillus, and Leuconostoc. Similar results were observed in MAL-ED participants (Peru), where Giardia Infection was associated with an enrichment of Prevotella and a reduction in Escherichia (Gammaproteobacteria; Berry et al., 2020). LEfSe also revealed an association between Giardia infection and an increase of Faecalibacterium, Blautia, Roseburia, Ruminococcus, and Ruminoccaceae, and an increase of Lachnospiraceae and Lactobacillus contrary to the GEMS study. Altogether these multicentered findings suggest that Giardia colonization is associated with a robust increase in Prevotella (enterotype II). Interestingly, Prevotella has previously been found to promote differentiation of Th17 cells (Calcinotto et al., 2018), which play a critical role in control of Giardia infection.

\section{Alterations to the Microbiome in Murine Models of Giardia Infection}

Giardia infection is associated with an altered gut microbiota composition throughout the gastrointestinal tract in mice (Figure 1.). A recent study showed that the microbial composition is altered at the phylum level (16S rRNA gene sequencing) upon infection with $G$. duodenalis isolate GS/M in both the small and large intestines, with a general increase in Proteobacteria and decrease in Firmicutes diversity in both antibiotic treated and antibiotic naive mice (Barash et al., 2017). In both antibiotic treated and antibiotic naive mice, significant decreases in Clostridia, a strict anaerobe, were observed throughout the small and large intestines. Strict aerobes including Rhodocyclaceae, Moraxellaceae, Flavobacteriales, and Comamonadaceae were enriched in the large and small intestines. Melainabacteria was found to be reduced in the proximal small intestines of antibiotic treated mice, but enriched in the distal small intestine. In contrast, in antibiotic naive mice Melainabacteria was reduced in both the proximal and distal small intestine. Taxa that were found to be enriched are generally considered to be metabolically flexible, and so will thrive under stress conditions induced by the presence of the pathogen, including arginine deprivation and altered nutrient availability (Barash et al., 2017). In a second study of G. duodenalis strain $\mathrm{H} 3$ infected mice, $16 \mathrm{~S}$ rRNA gene sequencing revealed a decrease in Bacteroidetes was observed in the upper small intestine while Firmicutes abundance remained relatively consistent compared to uninfected mice (Bartelt et al., 2017). Interestingly, a slight increase in OTUs for the mucolytic species Akkermansia was also observed (Bartelt et al., 2017). In a recent study using G. duodenalis strain GS/M (clone H7), greater microbiome shifts were observed in infected adult mice than in acutely infected neonates using $16 \mathrm{~S}$ sequencing. This was attributed both to the influence of mature vs. immature immune systems and to Giardia-induced enhancement of bile secretion in adult mice
(Riba et al., 2020). Indeed, populations of the bile salt deconjugating species Enterorhabdus were increased (Riba et al., 2020). In the large intestines, there was an overall increase in OTUs, and while the abundance of Staphylococcus decreased, increased populations of Coriobacteriaceae were observed (Riba et al., 2020).

Microbiota composition has also been investigated in immunocompromised animals (see section Microbiota-Immune System Interactions During Giardia Infection). In both IL-10 $0^{-/-}$ and $\mathrm{Tcra}^{-/-}$mice (animals deficient in alpha beta $\mathrm{T}$ cell receptor and devoid of CD4+ and CD8- and CD4- and CD8+ T cells), Giardia infection led to a significant reduction of microbial diversity (16S rRNA sequencing) that was not observed in wild-type mice. A reduction of Bacteroides and Firmicutes and an increase in Proteobacteria was evident, suggesting that regardless of different genetic backgrounds, mice with Giardia infections tend to develop microbiota dysbiosis (Dann et al., 2018). Expansion of Proteobacteria has previously been associated with intestinal inflammation and correlates with a disruption of the anaerobic state of the healthy colon (Litvak et al., 2017).

\section{Influence of Host Nutritional Status on Giardia-Microbiome Interactions}

Diet and the host nutritional status are known to play a key role during parasitic infections (Shea-Donohue et al., 2017). In giardiasis, micronutrient deficiencies, such as zinc, iron, and vitamin $\mathrm{A}$, are associated with increased host susceptibility to infection, impaired immune responses and intestinal malabsorption in young individuals (Duncombe et al., 1980; Astiazaran-Garcia et al., 2010, 2015; Quihui-Cota et al., 2012; Bartelt et al., 2013; Inigo-Figueroa et al., 2013). Supplementation of micronutrients and trace elements in children led to a reduction of the frequency and severity of diarrhea and a decrease in infection rate, indicating that diet intervention may be considered as an efficient therapeutic strategy (Lima et al., 2010; Astiazaran-Garcia et al., 2015). In mice, dietary fibers, dietary fat, protein deficiencies, and prebiotic fibers can modulate the outcome of giardiasis (by modulating parasite persistence, host immunity, intestinal barrier dysfunction, etc.); however, few studies have linked those effects to the gut microbiota (Upadhyay et al., 1985; Hamadto et al., 1989; Leitch et al., 1989; Magne et al., 1994; Rodrigues et al., 1994; Diaz-Cinco et al., 2002; Suh et al., 2004; Erlandsen, 2005; Gomes et al., 2012; Bartelt et al., 2013; Ventura et al., 2013; Shukla et al., 2016; Burgess et al., 2019). Recent evidence suggests that diet composition may at least in part modulate the microbiota dysbiosis associated with giardiasis. In mice fed a protein deficient diet (PD), both trophozoite burden and persistence have been shown to be increased compared to mice fed an isocaloric conventional diet (CD). Protein deficiency, in the absence of infection, causes significant dysbiosis in mice, as well as elevated bacterial abundance in the duodenum (Bartelt et al., 2017). Similarly, in Giardia-infected mice, distinct microbiome profiles are observed when comparing infected mice fed PD and CD. Greater duodenal bacterial abundance was observed via $16 \mathrm{~S}$ rRNA sequencing in G. duodenalis $\mathrm{H} 3$ infected PD mice compared to infected CD mice. Mice fed PD diets showed increased Firmicutes: Bacteroidetes ratios, 
with a significant increase in the abundance of Clostridiales. Giardia infection tended to further enhance Firmicutes abundance, in addition to reducing Bacteroidetes abundance (Bartelt et al., 2017). Giardia-induced growth impairment was enhanced by the PD diet and could be ameliorated by antibiotic treatment, suggesting an important role for the microbiota in the cooperative effects of Giardia infection and protein deficiency in growth impairment (Bartelt et al., 2017).

\section{Alterations to the Microbiome in Giardia-Infected Companion Animals}

Companion animals are frequent carriers of both symptomatic and asymptomatic Giardia infections (Figure 1; Tysnes et al., 2014; Bouzid et al., 2015; Stafford et al., 2020). The prevalence of giardiasis varies widely by region but has been estimated via meta-analysis of prevalence reports to be approximately $15 \%$ in dogs and $12 \%$ in cats (Bouzid et al., 2015). To date, only a handful of studies have characterized the gut microbiota of these infected animals. In free-roaming dogs, Giardia infection was associated with significant alteration of the fecal microbiome at the genus level, as analyzed through $16 \mathrm{~S}$ sequencing. Giardia positive groups showed an increase in Catenibacterium, Pseudomonas, and Howardella, while Giardia negative groups had higher levels of Bacteroides and Pseudobutyrivibrio (Slapeta et al., 2015). Domesticated dogs infected with Giardia in the United States similarly showed significant changes to fecal microbiome beta-diversity compared to uninfected animals (Berry et al., 2020). Of all the parasites studied (Giardia, Cystoisospora, hookworm, whipworm, ascaris, and tapeworm), Giardia alone, and Giardia with polymicrobial infections, caused the most significant difference in the beta diversity as compared to non-infected control animals. Specifically, Giardia was associated with an enrichment of Clostridium and Lactobacillus and a reduction in Bacteroides as determined by both Bray-Curtis and weighted UniFrac metrics, even when the confounding variables such as age and sex were controlled for. The distinct microbiome profile associated with Giardia positive samples was evident even in the context of co-infections and was observed with and without a history of antibiotic treatment or diarrheal symptoms (Berry et al., 2020). In fact, the Giardia co-infected animals were indistinguishable in terms of beta diversity from the animals that were infected with Giardia alone.

In Felidae, Giardia-infected cats, significant differences in overall microbiome profiles have yet to be documented; however, several genera appear to be altered in Giardia-positive cats, with increased Rosburia and decreased Subdoligranulum compared to Giardia-negative animals. In addition, the overall number of operational taxonomic units (OTUs) may be increased in Giardia infected cats (Slapeta et al., 2015).

\section{Giardia Trophozoites and Secretory- Excretory Products Promote Formation of Pathobionts}

As described above (section Structure and Composition of the Intestinal Microbiota), gut microbes can aggregate as a polymicrobial structure known as a biofilm, which (i) facilitates adhesion to their substrate, (ii) helps them to withstand shear forces (shear forces range from 0.02 to 35 dynes $/ \mathrm{cm}^{2}$ in force value in the gut) and (iii) increases their persistence and resistance in the gut through production of an extracellular matrix (ECM; Avvisato et al., 2007; Trujillo-de Santiago et al., 2018; Buret et al., 2019). To characterize the effects of Giardia on gut microbiota biofilms, a recent study examined interactions between $G$. duodenalis isolate NF and multi-species biofilms cultured from healthy human intestinal biopsies using the Calgary Biofilm Device (Beatty et al., 2017). Scanning electron and representative confocal scanning laser micrographs revealed that Giardia excretory/secretory products (ESPs) were able to actively degrade the ECM of human microbiota biofilms in a cysteine protease dependent manner, promoting increased dispersal of planktonic bacteria from the biofilm. When exposed to the intestinal epithelial cell line Caco-2, these dispersed planktonic bacteria demonstrated increased virulence compared to dispersed bacteria from control biofilms that had not been exposed to Giardia. These Giardia-induced pathobionts promoted the release of pro-inflammatory CXCL-8, in association with an upregulation of TLR4 pathway signaling (Beatty et al., 2017). Moreover, these bacteria degraded tight junctional proteins, such as ZO-1, and induced epithelial apoptosis, which together can promote bacterial translocation by reducing epithelial barrier function. To further characterize the effects of Giardia on microbiota biofilms, germ-free $\mathrm{C} 57 \mathrm{Bl} / 6$ mice were reconstituted with biofilm-dispersed planktonic bacteria obtained from Giardiaexposed or control biofilms. Histological examination revealed that Giardia-exposed bacteria-induced lymphocyte aggregation in the colonic mucosa and upregulated the pro-inflammatory cytokines IL- $1 \beta$, TNF- $\alpha$, IFN- $\gamma$, and IL-17 in colonic tissues 14 days post challenge compared with mice given control bacteria (Beatty et al., 2017). Mouse fecal microbiota composition (16s rRNA gene sequencing) in germ-free mice given Giardiatreated biofilm bacteria compared to those given untreated biofilm bacteria showed an overall increase in Firmicutes (Clostridiales) and Bacteroidetes at the phylum level. At the genus level, there was an increase of Sutterella, which is associated with interleukin secretion and colitis in humans, and a decrease of Parabacteroides and Phascolarctobacterium (Figure 1; Hiippala et al., 2016). Together, the data reveal a novel mechanism by which exposure to an enteropathogen, Giardia in this case, may drive pathogenesis by converting commensal biofilm microbes into pathobionts.

The ability of Giardia trophozoites to promote the release of pathobionts and alter bacterial virulence was further assessed in vivo using the nematode Caenorhabditis elegans as a model (Gerbaba et al., 2015, 2017). Alone, neither non-pathogenic E. coli strains OP50 or HB101 nor G. duodenalis isolate NF trophozoites had an effect on C. elegans viability. However, exposure to $E$. coli strain OP50 that had been grown in co-culture with G. duodenalis trophozoites or its ESPs resulted in the death of the nematode within $24 \mathrm{~h}$. Similar observations were made in C. elegans exposed to E. coli HB101 co-cultured with G. duodenalis (Gerbaba et al., 2015). Synergistic lethal effects of Giardia and E. coli were associated with facilitation of bacterial colonization of the C. elegans intestine. 
Transcriptomic analysis conducted in this study revealed that Giardia ESPs altered the expression of over 100 genes in E. coli HB101, including virulence factor genes, suggesting that Giardia-induced functional changes in commensal species can increase their toxicity to the host (Gerbaba et al., 2015). Additional experiments were conducted on human microbiota biofilms obtained from either healthy donors or from inflamed sites in patients with ulcerative colitis (UC). Upon prior exposure to $G$. duodenalis, both microbiota from healthy donors and UC patients became lethal toward C. elegans, while microbiota not exposed to Giardia showed little to no toxicity. Altogether, these findings indicate that Giardia can actively disrupt human commensal microbial biofilms and promote the release of pathobionts in the gut that may exert further pro-inflammatory effects. These effects may contribute at least in part to acute and post-infectious disease in giardiasis.

Additionally, recent studies have also elucidated that Giardia can release extracellular vesicles (EVs) that contain wellcharacterized virulence factors (Evans-Osses et al., 2015; Moyano et al., 2019; Gavinho et al., 2020). These EVs have been shown to play an important role in host-Giardia interaction. Whether Giardia EVs represent a potential mode of interaction between the parasite and the host's microbiota warrants further investigation.

\section{GIARDIASIS IN CO-INFECTIONS: A DOUBLE-EDGED SWORD}

\section{Prevalence of Enteropathogens in Concomitant Infection With Giardia in Humans}

Polymicrobial infections are defined as the presence of microorganisms that, under certain circumstances, can establish a concomitant infection (Brogden et al., 2005). These infections, mostly transmitted via the fecal-oral route, are very common in developing countries with poor sanitation and represent a burden on the development of children (Ryan and Caccio, 2013; Muhsen et al., 2014). Infection with G. duodenalis has been associated with concomitant infections with a variety of gut bacteria, viruses, and parasites. For example, in Venezuelan children, $62 \%$ with mild to moderate Ascaris lumbricoides infection were co-infected with G. duodenalis, and $45 \%$ of children with low A. lumbricoides burden were also infected with Giardia (Hagel et al., 2011). During an outbreak of cryptosporidiosis in a hospital ward in China, $6.3 \%$ of the patients infected with Cryptosporidium were also positive for G. duodenalis (Wang et al., 2013). Additionally, there has been a case of co-infection of G. duodenalis and Cyclospora cayetanensis in an immunocompetent patient with prolonged diarrhea in Turkey (Koru et al., 2006). Concomitant infection with C. difficile and G. duodenalis has also been reported (Khatami et al., 2004). In France, 4 of 25 patients diagnosed with Whipple disease were also infected with Giardia (Fenollar et al., 2003). In India, 32 and $19 \%$ of the diarrheal patients infected with Giardia were also coinfected with Vibrio cholera and rotavirus, respectively (Mukherjee et al., 2014). Concomitant infection of Giardia with E. coli, Campylobacter spp., Salmonella spp., and rotavirus are also frequent (Bilenko et al., 2004). Giardia has also been detected frequently in non-diarrhoeal stools along with Campylobacter spp., enteroaggregative E. coli, norovirus, LT-producing enterotoxigenic E coli (EPEC), and typical and atypical enteropathogenic E. coli strains (Platts-Mills et al., 2015). In Nicaragua, 70\% of G. duodenalis cases were co-infected with another enteropathogen, most commonly norovirus, sapovirus, or EPEC (Becker-Dreps et al., 2014). A recent report indicated that in a MAL-ED cohort, 14 of 215 Giardia infected children were also coinfected with either Cryptosporidum or rotavirus (Berry et al., 2020).

Several reports suggest that concomitant infections with $G$. duodenalis and Helicobacter pylori are frequent. In Ugandan children between 0 and 12 years of age, the incidence of Giardia infection was 3-fold higher (odds ratio of 2.9) in the presence of $H$. pylori compared to single-species infections (Ankarklev et al., 2012). In Egyptian children with recurrent abdominal pain (RAP), concomitant infection with $H$. pylori and Giardia was found in $40 \%$ of RAP patients compared to $12.2 \%$ in controls (Eldash et al., 2013). Similarly, prevalence of concomitant $H$. pylori infection and Giardia was $22.4 \%$ in RAP patients in Turkey, compared to $6.8 \%$ in the control groups (Zeyrek et al., 2008). In Italy, $H$. pylori was present in 37 of the 41 patients with gastric giardiasis (Doglioni et al., 1992). In Brazilian children, seropositivity to $H$. pylori antibodies was associated with the presence of Giardia with an odds ratio of 3.2 (Moreira et al., 2005). Gastric biopsy examination of patients with chronic cholecystitis associated with chronic gastroduodenitis revealed a high prevalence of co-infection with H. pylori and G. duodenalis (Isaeva and Efimova, 2010). Whether and how polyparasitism in giardiasis may contribute to disease is the topic of intense research activities.

\section{Giardia and Enteroaggregative Escherichia coli Synergize to Enhance Malnutrition and Growth Impairment by Inducing Metabolic Dysfunctions and Immune Perturbations}

In certain cases, co-infections with Giardia and other pathogens can result in negative outcomes for the host. Enteroaggregative Escherichia coli (EAEC) is one of the most common enteropathogens isolated from malnourished children, and as such is commonly found in Giardia co-infections (Platts-Mills et al., 2015). Using a murine model of protein malnutrition, recent findings observed that coinfection with $G$. duodenalis and EAEC promotes growth impairment, microbiota-dependent delayed parasite clearance, microbial metabolic perturbations in the gut, and an alteration of local host immune responses against EAEC (Bartelt et al., 2017). Combination of the two pathogens resulted in enhanced weight loss in mice fed a protein deficient (PD) diet compared to uninfected controls, whereas in mice fed with a conventional diet (CD), the infected groups showed no significant weight loss. These data provide compelling evidence that, in malnourished hosts, Giardia infection and polymicrobial infections may synergize to exacerbate stunted growth. In addition, in mice co-infected 
with EAEC, Giardia infection may cause extraintestinal manifestations by mediating and attenuating the cytokine response of bone marrow-derived dendritic cells (Burgess et al., 2019). A previous report had demonstrated growth impairment and decreased inflammatory responses in malnourished mice infected with Giardia alone (Bartelt et al., 2013). Together, these results are in accordance with epidemiological data showing that Giardia infection is more likely to contribute to decreased immune functions and growth impairment in malnourished children (Fink and Singer, 2017).

\section{Immunomodulation by Giardia Attenuates Enteropathogen-Induced Inflammation}

Evidence from epidemiological studies indicate that co-infections involving $G$. duodenalis may be associated with a protective effect against enteropathogen-driven diarrhea in developing countries (Bilenko et al., 2004; Veenemans et al., 2011; Muhsen et al., 2014; Cotton et al., 2015). An example of this protective effect comes from studies conducted on Bangladeshi and Tanzanian children, whereby children infected with Giardia present with reduced diarrheal illness as well as reduced serum inflammatory scores (Bilenko et al., 2004; Haque, 2007; Veenemans et al., 2011; Muhsen et al., 2014).

In an attempt to explain the mechanisms underlying the protective effects of Giardia against attaching and effacing pathogens (A/E pathogens), a murine model of coinfection with Giardia muris and the mouse enteropathogen Citrobacter rodentium was established (Manko et al., 2017). Results showed that $G$. muris can reduce the symptoms of $C$. rodentiuminduced colitis, including weight loss, intestinal permeability, and histopathological damages, by enhancing, at least in part, the production of mucosal antimicrobial peptides (AMPs) such as Mouse $\beta$-defensin 3 and Trefoil factor 3 (TFF3; Manko et al., 2017). Similarly, coinfection with G. duodenalis isolate NF and enteropathogenic E. coli (EPEC) of human intestinal epithelial cells results in cathepsin B-dependent increases in human $\beta$-defensin 2 and TFF3 gene expression, two major AMPs produced by intestinal epithelia (Manko et al., 2017). Elevated levels of MMP7, an intestinal matrix metalloprotease involved in $\alpha$-defensin production by Paneth cells, have been previously observed in response to Giardia infection in mice (Tako et al., 2013). In addition, Giardia trophozoites exert anti- EPEC effects ex-vivo in crypt killing assays (Manko et al., 2017). Recent research also explored the role of the NLRP3 inflammasome in the protective effects of Giardia during co-infections. The NLRP3 inflammasome is activated by other protozoan parasites such as Leishmania spp., Plasmodium spp., Entamoeba spp., Naegleria spp., Trypanosoma cruzi, and Toxoplasma gondii (Mortimer et al., 2014, 2015; Kim et al., 2016; de Carvalho et al., 2020). New findings now demonstrate that upregulation of mucosal AMPs and reduction of $C$. rodentium-induced colitis in mice co-infected with Giardia and C. rodentium is mediated by the activation of the NLRP3 inflammasome pathway (Manko-Prykhoda et al., 2020). In human intestinal epithelial cell lines, chemical inhibition of the NLRP3 inflammasome blocked the upregulation of AMP gene expression. Moreover, the antimicrobial activity of colonic crypt cells against A/E enteropathogens is enhanced by Giardia in a NLRP3-dependent manner (Manko-Prykhoda et al., 2020). The data uncover a novel protective role for NLRP3 during polyparasitism.

Other studies also investigated the immunomodulatory properties of Giardia against inflammatory responses to Clostridium difficile or Salmonella spp., two enteropathogens found concomitantly with Giardia (Bilenko et al., 2004; Khatami et al., 2004). In an in vivo model of $C$. difficile toxin $\mathrm{A}$ and B-induced colitis, G. duodenalis was shown to dampen granulocyte infiltration, decrease colonic myeloperoxidase activity (a marker of inflammation) and attenuate the expression and secretion of pro-inflammatory mediators and neutrophil chemoattractants, in a Giardia isolate-dependent manner (Cotton et al., 2014b). In intestinal epithelial cell cultures stimulated with Salmonella enterica (serovar Typhimurium), Giardia can actively degrade CXCL-8, a potent pro-inflammatory cytokine and neutrophil chemoattractant, in a cysteine protease-dependent fashion (Cotton et al., 2014a). Consistent with these observations, recent reports demonstrated that Giardia cysteine proteases can degrade immunoglobulins, chemokines and other cytokines, tight junctional proteins, and epithelial cytoskeletal villin (Bhargava et al., 2015; Liu et al., 2018, 2019; Ortega-Pierres et al., 2018; Allain et al., 2019). Together, these data further support the hypothesis that Giardia modulates the host response to enteropathogens during co-infections.

\section{POST-GIARDIASIS INTESTINAL DISORDERS: A ROLE FOR BACTERIAL TRANSLOCATION}

Irritable bowel syndrome (IBS) is a functional gastrointestinal disorder, characterized by recurring or chronic gastrointestinal symptoms including abdominal discomfort, altered gut motility, and visceral hypersensitivity (Simsek, 2011). One of the subtypes of IBS, post infectious irritable bowel syndrome (PI-IBS), is characterized by the manifestation of IBS symptoms following infections by enteric pathogens (Heitkemper et al., 2011). Recent research has demonstrated that Giardia infection can lead to the development of gastrointestinal disorders even after the successful clearance of the parasite. Indeed, Giardia infection can increase the risk of developing PI-IBS, and 5 to $10 \%$ of patients with IBS have been previously infected with Giardia (Horman et al., 2004a,b; Grazioli et al., 2006; Hanevik et al., 2009; Dizdar et al., 2010; Allain and Buret, 2020). In a cohort study conducted following the outbreak of giardiasis in Bergen, Norway, $80.5 \%$ of patients developed symptoms consistent with PI-IBS (Hanevik et al., 2009). However, the mechanisms responsible for this phenomenon warrant further investigation. To establish the causality between Giardia and PI-IBS, a novel neonatal rat model of intestinal hypersensitivity was developed (Halliez et al., 2016). In this model, 50 days post-infection, after full clearance of the parasite, significantly increased jejunal and rectal visceral hypersensitivity was observed. Upregulation of $c$-fos gene expression, a marker of neuronal activation, was clearly evident in post-clearance infected animals. 
One factor underlying visceral hypersensitivity is an increase in intestinal permeability (Zhou et al., 2009). Degradation and rearrangement of tight junctional proteins such as Zo-1, claudin-1, and claudin-4 is a hallmark of Giardia infection (Allain and Buret, 2020). Paracellular bacterial translocation is observed in giardiasis during the acute phase of infection and persists during the post-infectious state (Halliez et al., 2016). Previous research also showed that Giardia infection in mice causes persistent tight junctional damage and bacterial translocation during the acute phase and after trophozoite clearance (postinfective phase; Chen et al., 2013). Bacterial translocation was associated with increased neutrophil infiltration, increased myeloperoxidase activity, and elevated mucosal levels of pro-inflammatory cytokines such as IFN $\gamma$, TNF $\alpha$, and IL- $1 \beta$, during both the acute and post-infective phases (Chen et al., 2013). In addition, alterations to the mucus barrier as a result of mucin degradation and altered mucus production and secretion during infection may further enhance barrier dysfunction (Amat et al., 2017) Thus, bacteria-driven inflammation may be perpetuated even after parasite clearance, which may contribute to development of post-infectious intestinal disorders (Chen et al., 2013; Singer et al., 2019).

Small intestinal bacterial overgrowth (SIBO) is another hallmark of IBS (Ghoshal and Gwee, 2017). Duodenal bacterial overgrowth in association with bile salt deconjugation has been reported in subjects with symptomatic giardiasis, possibly leading to fat malabsorption (Tandon et al., 1977; Muller and von Allmen, 2005). In an attempt to correlate SIBO to post-infectious giardiasis, patients with gastrointestinal disorders following a Giardia outbreak were tested with a lactulose breath test, which provides a measurement of hydrogen and methane levels. This study failed to show differences between patients, indicating that giardiasis may not necessarily be associated with SIBO (Morken et al., 2008).

\section{CONCLUSION}

Disruption of the host intestinal microbiome is well documented in a variety of enteropathogenic infections, and recent evidence has indicated critical roles for the microbiome in modulating disease outcome. During G. duodenalis infections, alterations to the composition and functions of the host commensal microbiota have been demonstrated, including an alteration

\section{REFERENCES}

Ahmed, S., Macfarlane, G. T., Fite, A., Mcbain, A. J., Gilbert, P., and Macfarlane, S. (2007). Mucosa-associated bacterial diversity in relation to human terminal ileum and colonic biopsy samples. Appl. Environ. Microbiol. 73, 7435-7442. doi: 10.1128/AEM.01143-07

Alazzaz, J., Chaouch, S., Boucard, A. S., Bermudez-Humaran, L. G., and Florent, I. (2020). "Probiotics as anti-Giardia defenders: overview on putative control mechanisms" in Eukaryome impact on human intestine homeostasis and mucosal immunology. ed. N. Guillen (Cham: Springer), 335-349.

Aley, S. B., Zimmerman, M., Hetsko, M., Selsted, M. E., and Gillin, F. D. (1994). Killing of Giardia lamblia by cryptdins and cationic neutrophil peptides. Infect. Immun. 62, 5397-5403. doi: 10.1128/IAI.62.12.5397-5403.1994 of bacterial species composition and diversity, as well as a direct disruption of intestinal bacterial biofilm structure, which can be associated with the release of pathobionts. Important discoveries on the role of the intestinal microbiota in Giardia pathogenesis have been made through the use of germ-free and antibiotic-treated animal models. In addition, by modulating host immunity, mucosal cell function, and the intestinal microenvironment, the microbiome has been shown to be a determining factor in susceptibility or resistance to Giardia infection and in disease severity and duration. The development of gnotobiotic animal models will aim to identify the specific roles of individual commensal species in giardiasis, which may represent future therapeutic targets. In addition, microbiota disruption may contribute to development of post-infectious complications including PI-IBS. Further investigation is required to determine how the disruption of homeostatic interactions between microbes and host cells may contribute to chronic intestinal and extraintestinal complications during giardiasis.

Finally, dietary interventions and the use of probiotics are emerging as potential treatment options for giardiasis as well as other GI diseases in which the gut microbiota is actively implicated. Probiotic microbes may have direct or indirect anti-parasitic effects in the gut and have been shown to reduce the severity and duration of Giardia infection. Diet may represent a novel risk factor for development of severe giardiasis and for development of post-infectious complications, particularly in malnourished children. Diet has been shown to influence gut microbiota composition, which may explain in part population specific symptom variability. Future clinical research will be required to determine the efficacy of dietary and probiotic interventions and to identify populations in which these interventions will be most effective. Because Giardia has been shown to have both protective and detrimental effects on overall health in human populations and animal models, it will be important to further our understanding of how the microbiome contributes to variations in clinical manifestations.

\section{AUTHOR CONTRIBUTIONS}

EF, TA, AS, OS, and AB: contribution to manuscript design and writing. All authors contributed to the article and approved the submitted version.

Allain, T., and Buret, A. G. (2020). Pathogenesis and post-infectious complications in giardiasis. Adv. Parasitol. 107, 173-199. doi: 10.1016/bs.apar.2019. 12.001

Allain, T., Chaouch, S., Thomas, M., Travers, M. A., Valle, I., Langella, P., et al. (2018a). Bile salt hydrolase activities: a novel target to screen antiGiardia lactobacilli? Front. Microbiol. 9:89. doi: 10.3389/fmicb.2018.00089

Allain, T., Chaouch, S., Thomas, M., Vallee, I., Buret, A. G., Langella, P., et al. (2017). Bile-salt-hydrolases from the probiotic strain Lactobacillus johnsonii La1 mediate anti-giardial activity in vitro and in vivo. Front. Microbiol. 8:2707. doi: 10.3389/fmicb.2017.02707

Allain, T., Fekete, E., and Buret, A. G. (2019). Giardia cysteine proteases: the teeth behind the smile. Trends Parasitol. 35, 636-648. doi: 10.1016/j. pt.2019.06.003 
Allain, T., Groves, R., Lewis, I. A., and Buret, A. G. (2018b). The metabolomic signature of Giardia sp. FASEB J. 32:406.403. doi: 10.1096/fasebj.2018.32.1_ supplement.406.3

Amat, C. B., Motta, J. P., Fekete, E., Moreau, F., Chadee, K., and Buret, A. G. (2017). Cysteine protease-dependent mucous disruptions and differential mucin gene expression in Giardia duodenalis infection. Am. J. Pathol. 187, 2486-2498. doi: 10.1016/j.ajpath.2017.07.009

Amer, E. I., Mossallam, S. F., and Mahrous, H. (2014). Therapeutic enhancement of newly derived bacteriocins against Giardia lamblia. Exp. Parasitol. 146, 52-63. doi: 10.1016/j.exppara.2014.09.005

Ankarklev, J., Hestvik, E., Lebbad, M., Lindh, J., Kaddu-Mulindwa, D. H., Andersson, J. O., et al. (2012). Common coinfections of Giardia intestinalis and Helicobacter pylori in non-symptomatic Ugandan children. PLoS Negl. Trop. Dis. 6:e1780. doi: 10.1371/journal.pntd.0001780

Astiazaran-Garcia, H., Inigo-Figueroa, G., Quihui-Cota, L., and Anduro-Corona, I. (2015). Crosstalk between zinc status and Giardia infection: a new approach. Nutrients 7, 4438-4452. doi: 10.3390/nu7064438

Astiazaran-Garcia, H., Lopez-Teros, V., Valencia, M. E., Vazquez-Ortiz, F., Sotelo-Cruz, N., and Quihui-Cota, L. (2010). Giardia lamblia infection and its implications for vitamin A liver stores in school children. Ann. Nutr. Metab. 57, 228-233. doi: 10.1159/000321682

Atarashi, K., Tanoue, T., Ando, M., Kamada, N., Nagano, Y., Narushima, S., et al. (2015). Th17 cell induction by adhesion of microbes to intestinal epithelial cells. Cell 163, 367-380. doi: 10.1016/j.cell.2015.08.058

Audebert, C., Even, G., Cian, A., Blastocystis Investigation Group, Loywick, A., Merlin, S., et al. (2016). Colonization with the enteric protozoa Blastocystis is associated with increased diversity of human gut bacterial microbiota. Sci. Rep. 6:25255. doi: 10.1038/srep25255

Avvisato, C. L., Yang, X., Shah, S., Hoxter, B., Li, W., Gaynor, R., et al. (2007). Mechanical force modulates global gene expression and beta-catenin signaling in colon cancer cells. J. Cell Sci. 120, 2672-2682. doi: 10.1242/jcs. 03476

Barash, N. R., Maloney, J. G., Singer, S. M., and Dawson, S. C. (2017). Giardia alters commensal microbial diversity throughout the murine gut. Infect. Immun. 85, e00948-e009416. doi: 10.1128/IAI.00948-16

Bartelt, L. A., Bolick, D. T., Mayneris-Perxachs, J., Kolling, G. L., Medlock, G. L., Zaenker, E. I., et al. (2017). Cross-modulation of pathogen-specific pathways enhances malnutrition during enteric co-infection with Giardia lamblia and enteroaggregative Escherichia coli. PLoS Pathog. 13:e1006471. doi: 10.1371/ journal.ppat.1006471

Bartelt, L. A., Roche, J., Kolling, G., Bolick, D., Noronha, F., Naylor, C., et al. (2013). Persistent G. lamblia impairs growth in a murine malnutrition model. J. Clin. Invest. 123, 2672-2684. doi: 10.1172/JCI67294

Baum, K. F., Berens, R. L., and Marr, J. J. (1993). Purine nucleoside and nucleobase cell membrane transport in Giardia lamblia. J. Eukaryot. Microbiol. 40, 643-649. doi: 10.1111/j.1550-7408.1993.tb06122.x

Beatty, J. K., Akierman, S. V., Motta, J. P., Muise, S., Workentine, M. L., Harrison, J. J., et al. (2017). Giardia duodenalis induces pathogenic dysbiosis of human intestinal microbiota biofilms. Int. J. Parasitol. 47, 311-326. doi: 10.1016/j.ijpara.2016.11.010

Becker-Dreps, S., Bucardo, F., Vilchez, S., Zambrana, L. E., Liu, L., Weber, D. J., et al. (2014). Etiology of childhood diarrhea after rotavirus vaccine introduction: a prospective, population-based study in Nicaragua. Pediatr. Infect. Dis. J. 33, 1156-1163. doi: 10.1097/INF.0000000000000427

Belosevic, M., and Daniels, C. W. (1992). Phagocytosis of Giardia lamblia trophozoites by cytokine-activated macrophages. Clin. Exp. Immunol. 87, 304-309. doi: 10.1111/j.1365-2249.1992.tb02992.x

Benyacoub, J., Perez, P. F., Rochat, F., Saudan, K. Y., Reuteler, G., Antille, N., et al. (2005). Enterococcus faecium SF68 enhances the immune response to Giardia intestinalis in mice. J. Nutr. 135, 1171-1176. doi: 10.1093/jn/135.5.1171

Berry, A. S. F., Johnson, K., Martins, R., Sullivan, M. C., Farias Amorim, C., Putre, A., et al. (2020). Natural infection with Giardia is associated with altered community structure of the human and canine gut microbiome. mSphere 5, e00670-e006720. doi: 10.1128/mSphere.00670-20

Besirbellioglu, B. A., Ulcay, A., Can, M., Erdem, H., Tanyuksel, M., Avci, I. Y., et al. (2006). Saccharomyces boulardii and infection due to Giardia lamblia. Scand. J. Infect. Dis. 38, 479-481. doi: 10.1080/00365540600561769

Bhargava, A., Cotton, J. A., Dixon, B. R., Gedamu, L., Yates, R. M., and Buret, A. G. (2015). Giardia duodenalis surface cysteine proteases induce cleavage of the intestinal epithelial cytoskeletal protein villin via myosin light chain kinase. PLoS One 10:e0136102. doi: 10.1371/journal.pone.0136102

Bienz, M., Dai, W. J., Welle, M., Gottstein, B., and Muller, N. (2003). Interleukin-6deficient mice are highly susceptible to Giardia lamblia infection but exhibit normal intestinal immunoglobulin A responses against the parasite. Infect. Immun. 71, 1569-1573. doi: 10.1128/iai.71.3.1569-1573.2003

Bilenko, N., Levy, A., Dagan, R., Deckelbaum, R. J., El-On, Y., and Fraser, D. (2004). Does co-infection with Giardia lamblia modulate the clinical characteristics of enteric infections in young children? Eur. J. Epidemiol. 19, 877-883. doi: 10.1023/b:ejep.0000040533.75646.9c

Bouzid, M., Halai, K., Jeffreys, D., and Hunter, P. R. (2015). The prevalence of Giardia infection in dogs and cats, a systematic review and meta-analysis of prevalence studies from stool samples. Vet. Parasitol. 207, 181-202. doi: 10.1016/j.vetpar.2014.12.011

Brogden, K. A., Guthmiller, J. M., and Taylor, C. E. (2005). Human polymicrobial infections. Lancet 365, 253-255. doi: 10.1016/S0140-6736(05)17745-9

Buret, A., Hardin, J. A., Olson, M. E., and Gall, D. G. (1992). Pathophysiology of small intestinal malabsorption in gerbils infected with Giardia lamblia. Gastroenterology 103, 506-513. doi: 10.1016/0016-5085(92)90840-u

Buret, A. G., Motta, J. P., Allain, T., Ferraz, J., and Wallace, J. L. (2019). Pathobiont release from dysbiotic gut microbiota biofilms in intestinal inflammatory diseases: a role for iron? J. Biomed. Sci. 26:1. doi: 10.1186/s12929-018-0495-4

Burgess, S. L., Gilchrist, C. A., Lynn, T. C., and Petri, W. A. Jr. (2017). Parasitic protozoa and interactions with the host intestinal microbiota. Infect. Immun. 85, e00101-e001017. doi: 10.1128/IAI.00101-17

Burgess, S. L., Oka, A., Liu, B., Bolick, D. T., Oakland, D. N., Guerrant, R. L., et al. (2019). Intestinal parasitic infection alters bone marrow derived dendritic cell inflammatory cytokine production in response to bacterial endotoxin in a diet-dependent manner. PLoS Negl. Trop. Dis. 13:e0007515. doi: 10.1371/ journal.pntd.0007515

Burmolle, M., Ren, D., Bjarnsholt, T., and Sorensen, S. J. (2014). Interactions in multispecies biofilms: do they actually matter? Trends Microbiol. 22, 84-91. doi: 10.1016/j.tim.2013.12.004

Calcinotto, A., Brevi, A., Chesi, M., Ferrarese, R., Garcia Perez, L., Grioni, M., et al. (2018). Microbiota-driven interleukin-17-producing cells and eosinophils synergize to accelerate multiple myeloma progression. Nat. Commun. 9:4832. doi: 10.1038/s41467-018-07305-8

Carding, S., Verbeke, K., Vipond, D. T., Corfe, B. M., and Owen, L. J. (2015). Dysbiosis of the gut microbiota in disease. Microb. Ecol. Health Dis. 26:26191. doi: 10.3402/mehd.v26.26191

Cascais-Figueiredo, T., Austriaco-Teixeira, P., Fantinatti, M., Silva-Freitas, M. L., Santos-Oliveira, J. R., Coelho, C. H., et al. (2019). Giardiasis alters intestinal fatty acid binding protein (I-FABP) and plasma cytokines levels in children in Brazil. Pathogens 9:7. doi: 10.3390/pathogens9010007

Charania, R., Wade, B. E., Mcnair, N. N., and Mead, J. R. (2020). Changes in the microbiome of cryptosporidium-infected mice correlate to differences in susceptibility and infection levels. Microorganisms 8:879. doi: 10.3390/ microorganisms 8060879

Chen, T. L., Chen, S., Wu, H. W., Lee, T. C., Lu, Y. Z., Wu, L. L., et al. (2013). Persistent gut barrier damage and commensal bacterial influx following eradication of Giardia infection in mice. Gut Pathog. 5:26. doi: 10.1186/1757-4749-5-26

Cornick, S., Tawiah, A., and Chadee, K. (2015). Roles and regulation of the mucus barrier in the gut. Tissue Barriers 3:e982426. doi: 10.4161/21688370.2014.982426

Cotton, J. A., Amat, C. B., and Buret, A. G. (2015). Disruptions of host immunity and inflammation by Giardia duodenalis: potential consequences for coinfections in the gastro-intestinal tract. Pathogens 4, 764-792. doi: 10.3390/ pathogens 4040764

Cotton, J. A., Bhargava, A., Ferraz, J. G., Yates, R. M., Beck, P. L., and Buret, A. G. (2014a). Giardia duodenalis cathepsin B proteases degrade intestinal epithelial interleukin-8 and attenuate interleukin-8-induced neutrophil chemotaxis. Infect. Immun. 82, 2772-2787. doi: 10.1128/IAI.01771-14

Cotton, J. A., Motta, J. P., Schenck, L. P., Hirota, S. A., Beck, P. L., and Buret, A. G. (2014b). Giardia duodenalis infection reduces granulocyte infiltration in an in vivo model of bacterial toxin-induced colitis and attenuates inflammation in human intestinal tissue. PLoS One 9:e109087. doi: 10.1371/ journal.pone.0109087

Dann, S. M., Le, C. H. Y., Hanson, E. M., Ross, M. C., and Eckmann, L. (2018). Giardia infection of the small intestine induces chronic colitis in genetically susceptible hosts. J. Immunol. 201, 548-559. doi: 10.4049/jimmunol.1700824 
Dann, S. M., Manthey, C. F., Le, C., Miyamoto, Y., Gima, L., Abrahim, A., et al. (2015). IL-17A promotes protective IgA responses and expression of other potential effectors against the lumen-dwelling enteric parasite Giardia. Exp. Parasitol. 156, 68-78. doi: 10.1016/j.exppara.2015.06.003

de Carvalho, R. V. H., Lima-Junior, D. S., Da Silva, M. V. G., Dilucca, M., Rodrigues, T. S., Horta, C. V., et al. (2020). Publisher correction: Leishmania RNA virus exacerbates Leishmaniasis by subverting innate immunity via TLR3-mediated NLRP3 inflammasome inhibition. Nat. Commun. 11:203. doi: 10.1038/s41467-019-13900-0

de Vos, W. M. (2015). Microbial biofilms and the human intestinal microbiome. NPJ Biofilms Microbiomes 1:15005. doi: 10.1038/npjbiofilms.2015.5

Diaz-Cinco, M. E., Ballesteros-Vazquez, M. N., Perez-Morales, R., and Mata-Haro, V. (2002). Impact of diet on the induction of infection with Giardia lamblia cysts in Sprague-Dawley rats. Salud Publica Mex. 44, 315-322.

Dizdar, V., Spiller, R., Singh, G., Hanevik, K., Gilja, O. H., El-Salhy, M., et al. (2010). Relative importance of abnormalities of CCK and 5-HT (serotonin) in Giardia-induced post-infectious irritable bowel syndrome and functional dyspepsia. Aliment. Pharmacol. Ther. 31, 883-891. doi: 10.1111/j.1365-2036.2010.04251.x

Doglioni, C., De Boni, M., Cielo, R., Laurino, L., Pelosio, P., Braidotti, P., et al. (1992). Gastric giardiasis. J. Clin. Pathol. 45, 964-967. doi: 10.1136/jcp.45.11.964

Dreesen, L., De Bosscher, K., Grit, G., Staels, B., Lubberts, E., Bauge, E., et al. (2014). Giardia muris infection in mice is associated with a protective interleukin 17A response and induction of peroxisome proliferator-activated receptor alpha. Infect. Immun. 82, 3333-3340. doi: 10.1128/IAI.01536-14

Duncombe, V. M., Bolin, T. D., Davis, M., Fagan, M. R., and Davis, A. E. (1980). The effect of iron deficiency, protein deficiency and dexamethasone on infection, re-infection and treatment of Giardia muris in the mouse. Aust. J. Exp. Biol. Med. Sci. 58, 19-26. doi: 10.1038/icb.1980.2

Dunn, N., and Juergens, A. L. (2020). "Giardiasis" in StatPearls. Treasure Island, FL: StatPearls Publishing.

El-Gebaly, N. S., Halawa, E. F., Moussa, H. M., Rabia, I., and Abu-Zekry, M. (2012). Saliva and sera IgA and IgG in Egyptian Giardia-infected children. Parasitol. Res. 111, 571-575. doi: 10.1007/s00436-012-2869-y

Eldash, H. H., Bekhit, O. E., and Algameel, A. A. (2013). Impact of Helicobacter pylori-giardiasis coinfection on children with recurrent abdominal pain. J. Egypt. Soc. Parasitol. 43, 509-516. doi: 10.12816/0006407

Ericsson, A. C., Hagan, C. E., Davis, D. J., and Franklin, C. L. (2014). Segmented filamentous bacteria: commensal microbes with potential effects on research. Comp. Med. 64, 90-98.

Erlandsen, S. (2005). Reduction in fecal excretion of Giardia cysts: effect of cholestasis and diet. J. Parasitol. 91, 1482-1484. doi: 10.1645/GE-3497RN.1

Evans-Osses, I., Reichembach, L. H., and Ramirez, M. I. (2015). Exosomes or microvesicles? Two kinds of extracellular vesicles with different routes to modify protozoan-host cell interaction. Parasitol. Res. 114, 3567-3575. doi: 10.1007/s00436-015-4659-9

Fekete, E., Amat, C., Allain, T., Mihara, K., Hollenberg, M., Chadee, K., et al. (2019). Giardia-induced alterations to intestinal mucus production involve protease-activated receptor-2-mediated activation of MAPK and calcium release. FASEB J. 33:38.10. doi: 10.1096/fasebj.2019.33.1_supplement.38.10

Fenimore, A., Martin, L., and Lappin, M. R. (2017). Evaluation of metronidazole with and without Enterococcus faecium SF68 in shelter dogs with diarrhea. Top. Companion Anim. Med. 32, 100-103. doi: 10.1053/j.tcam.2017.11.001

Fenollar, F., Lepidi, H., Gerolami, R., Drancourt, M., and Raoult, D. (2003). Whipple disease associated with giardiasis. J. Infect. Dis. 188, 828-834. doi: 10.1086/378093

Figueroa-Gonzalez, I., Quijano, G., Ramirez, G., and Cruz-Guerrero, A. (2011). Probiotics and prebiotics--perspectives and challenges. J. Sci. Food Agric. 91, 1341-1348. doi: 10.1002/jsfa.4367

Fink, M. Y., Maloney, J., Keselman, A., Li, E., Menegas, S., Staniorski, C., et al. (2019). Proliferation of resident macrophages is dispensable for protection during Giardia duodenalis infections. Immunohorizons 3, 412-421. doi: 10.4049/immunohorizons.1900041

Fink, M. Y., and Singer, S. M. (2017). The intersection of immune responses, microbiota, and pathogenesis in giardiasis. Trends Parasitol. 33, 901-913. doi: $10.1016 /$ j.pt.2017.08.001

Flynn, K. J., Ruffin, M. T. T., Turgeon, D. K., and Schloss, P. D. (2018). Spatial variation of the native colon microbiota in healthy adults. Cancer Prev. Res. 11, 393-402. doi: 10.1158/1940-6207.CAPR-17-0370

Fonseca, J. F., Alvim, L. B., Nunes, A. C., Oliveira, F. M. S., Amaral, R. S., Caliari, M. V., et al. (2019). Probiotic effect of Bifidobacterium longum 5(1A) and Weissella paramesenteroides WpK4 on gerbils infected with Giardia lamblia. J. Appl. Microbiol. 127, 1184-1191. doi: 10.1111/jam.14338

Franco, M. C., Golowczyc, M. A., De Antoni, G. L., Perez, P. F., Humen, M., and Serradell, M. L. A. (2013). Administration of kefir-fermented milk protects mice against Giardia intestinalis infection. J. Med. Microbiol. 62, 1815-1822. doi: 10.1099/jmm.0.068064-0

Garland, C. D., Lee, A., and Dickson, M. R. (1982). Segmented filamentous bacteria in the rodent small intestine: their colonization of growing animals and possible role in host resistance to Salmonella. Microb. Ecol. 8, 181-190. doi: 10.1007/BF02010451

Gavinho, B., Sabatke, B., Feijoli, V., Rossi, I. V., Da Silva, J. M., Evans-Osses, I., et al. (2020). Peptidylarginine deiminase inhibition abolishes the production of large extracellular vesicles from Giardia intestinalis, affecting host-pathogen interactions by hindering adhesion to host cells. Front. Cell. Infect. Microbiol. 10:417. doi: 10.3389/fcimb.2020.00417

Gerbaba, T. K., Green-Harrison, L., and Buret, A. G. (2017). Modeling hostmicrobiome interactions in Caenorhabditis elegans. J. Nematol. 49, 348-356.

Gerbaba, T. K., Gupta, P., Rioux, K., Hansen, D., and Buret, A. G. (2015). Giardia duodenalis-induced alterations of commensal bacteria kill Caenorhabditis elegans: a new model to study microbial-microbial interactions in the gut. Am. J. Physiol. Gastrointest. Liver Physiol. 308, G550-G561. doi: 10.1152/ajpgi.00335.2014

Ghoshal, U. C., and Gwee, K. A. (2017). Post-infectious IBS, tropical sprue and small intestinal bacterial overgrowth: the missing link. Nat. Rev. Gastroenterol. Hepatol. 14, 435-441. doi: 10.1038/nrgastro.2017.37

Gomes, M. A., De Oliveira, D. R., De Freitas, S. E., De Pinho Viana, M., and Borges, E. L. (2012). Effect of giardiasis combined with low-protein diet on intestinal absorption of glucose and electrolytes in gerbils. Exp. Parasitol. 131, 448-451. doi: 10.1016/j.exppara.2012.04.016

Goyal, N., Rishi, P., and Shukla, G. (2013). Lactobacillus rhamnosus GG antagonizes Giardia intestinalis induced oxidative stress and intestinal disaccharidases: an experimental study. World J. Microbiol. Biotechnol. 29, 1049-1057. doi: 10.1007/s11274-013-1268-6

Goyal, N., and Shukla, G. (2013). Probiotic Lactobacillus rhamnosus GG modulates the mucosal immune response in Giardia intestinalis-infected BALB/c mice. Dig. Dis. Sci. 58, 1218-1225. doi: 10.1007/s10620-012-2503-y

Goyal, N., Tiwari, R. P., and Shukla, G. (2011). Lactobacillus rhamnosus GG as an effective probiotic for murine giardiasis. Interdiscip. Perspect. Infect. Dis. 2011:795219. doi: 10.1155/2011/795219

Grazioli, B., Matera, G., Laratta, C., Schipani, G., Guarnieri, G., Spiniello, E., et al. (2006). Giardia lamblia infection in patients with irritable bowel syndrome and dyspepsia: a prospective study. World J. Gastroenterol. 12, 1941-1944. doi: 10.3748/wjg.v12.i12.1941

Guarner, F., and Malagelada, J. R. (2003). Gut flora in health and disease. Lancet 361, 512-519. doi: 10.1016/S0140-6736(03)12489-0

Hagel, I., Cabrera, M., Puccio, F., Santaella, C., Buvat, E., Infante, B., et al. (2011). Co-infection with Ascaris lumbricoides modulates protective immune responses against Giardia duodenalis in school Venezuelan rural children. Acta Trop. 117, 189-195. doi: 10.1016/j.actatropica.2010.12.001

Halliez, M. C., and Buret, A. G. (2013). Extra-intestinal and long term consequences of Giardia duodenalis infections. World J. Gastroenterol. 19, 8974-8985. doi: 10.3748/wjg.v19.i47.8974

Halliez, M. C., Motta, J. P., Feener, T. D., Guerin, G., Legoff, L., Francois, A., et al. (2016). Giardia duodenalis induces paracellular bacterial translocation and causes postinfectious visceral hypersensitivity. Am. J. Physiol. Gastrointest. Liver Physiol. 310, G574-G585. doi: 10.1152/ajpgi.00144.2015

Hamad, I., Raoult, D., and Bittar, F. (2016). Repertory of eukaryotes (eukaryome) in the human gastrointestinal tract: taxonomy and detection methods. Parasite Immunol. 38, 12-36. doi: 10.1111/pim.12284

Hamadto, H. H., Rashad, S. M., El-Ridi, A. M., Amin, F. A., Lotfy, N. A., Darwish, S. A., et al. (1989). Effect of diet on experimental giardiasis. J. Egypt. Soc. Parasitol. 19, 573-581.

Hanevik, K., Dizdar, V., Langeland, N., and Hausken, T. (2009). Development of functional gastrointestinal disorders after Giardia lamblia infection. BMC Gastroenterol. 9:27. doi: 10.1186/1471-230X-9-27

Haque, R. (2007). Human intestinal parasites. J. Health Popul. Nutr. 25, 387-391. Harvey, T. V., Tang, A. M., Da Paixao Seva, A., Albano Dos Santos, C., Santos Carvalho, S. M., Magalhaes Da Rocha, C. M. B., et al. (2020). Enteric parasitic infections in children and dogs in resource-poor communities in 
northeastern Brazil: identifying priority prevention and control areas. PLoS Negl. Trop. Dis. 14:e0008378. doi: 10.1371/journal.pntd.0008378

Hayashi, H., Takahashi, R., Nishi, T., Sakamoto, M., and Benno, Y. (2005). Molecular analysis of jejunal, ileal, caecal and recto-sigmoidal human colonic microbiota using $16 \mathrm{~S}$ rRNA gene libraries and terminal restriction fragment length polymorphism. J. Med. Microbiol. 54, 1093-1101. doi: 10.1099/jmm.0.45935-0 Heitkemper, M., Cain, K. C., Shulman, R., Burr, R., Poppe, A., and Jarrett, M. (2011). Subtypes of irritable bowel syndrome based on abdominal pain/ discomfort severity and bowel pattern. Dig. Dis. Sci. 56, 2050-2058. doi: 10.1007/s10620-011-1567-4

Hiippala, K., Kainulainen, V., Kalliomaki, M., Arkkila, P., and Satokari, R. (2016). Mucosal prevalence and interactions with the epithelium indicate commensalism of Sutterella spp. Front. Microbiol. 7:1706. doi: 10.3389/ fmicb.2016.01706

Hill, C., Guarner, F., Reid, G., Gibson, G. R., Merenstein, D. J., Pot, B., et al. (2014). Expert consensus document. The International Scientific Association for Probiotics and Prebiotics consensus statement on the scope and appropriate use of the term probiotic. Nat. Rev. Gastroenterol. Hepatol. 11, 506-514. doi: $10.1038 /$ nrgastro.2014.66

Hill, D. R., Guerrant, R. L., Pearson, R. D., and Hewlett, E. L. (1983). Giardia lamblia infection of suckling mice. J. Infect. Dis. 147, 217-221. doi: 10.1093/ infdis/147.2.217

Hjollo, T., Bratland, E., Steinsland, H., Radunovic, M., Langeland, N., and Hanevik, K. (2018). Longitudinal cohort study of serum antibody responses towards Giardia lamblia variant-specific surface proteins in a non-endemic area. Exp. Parasitol. 191, 66-72. doi: 10.1016/j.exppara.2018.06.005

Hollister, E. B., Gao, C., and Versalovic, J. (2014). Compositional and functional features of the gastrointestinal microbiome and their effects on human health. Gastroenterology 146, 1449-1458. doi: 10.1053/j.gastro.2014.01.052

Horman, A., Korpela, H., Sutinen, J., Wedel, H., and Hanninen, M. L. (2004a). Meta-analysis in assessment of the prevalence and annual incidence of Giardia spp. and Cryptosporidium spp. infections in humans in the Nordic countries. Int. J. Parasitol. 34, 1337-1346. doi: 10.1016/j.jpara.2004.08.009

Horman, A., Rimhanen-Finne, R., Maunula, L., Von Bonsdorff, C. H., Torvela, N., Heikinheimo, A., et al. (2004b). Campylobacter spp., Giardia spp., Cryptosporidium spp., noroviruses, and indicator organisms in surface water in southwestern Finland, 2000-2001. Appl. Environ. Microbiol. 70, 87-95. doi: 10.1128 /aem.70.1.87-95.2004

Humen, M. A., De Antoni, G. L., Benyacoub, J., Costas, M. E., Cardozo, M. I., Kozubsky, L., et al. (2005). Lactobacillus johnsonii Lal antagonizes Giardia intestinalis in vivo. Infect. Immun. 73, 1265-1269. doi: 10.1128/ IAI.73.2.1265-1269.2005

Iacob, S., and Iacob, D. G. (2019). Infectious threats, the intestinal barrier, and its trojan horse: dysbiosis. Front. Microbiol. 10:1676. doi: 10.3389/fmicb. 2019.01676

Iebba, V., Santangelo, F., Totino, V., Pantanella, F., Monsia, A., Di Cristanziano, V., et al. (2016). Gut microbiota related to Giardia duodenalis, Entamoeba spp. and Blastocystis hominis infections in humans from Côte d'Ivoire. J. Infect. Dev. Ctries 10, 1035-1041. doi: 10.3855/jidc.8179

Inigo-Figueroa, G., Mendez-Estrada, R. O., Quihui-Cota, L., Velasquez-Contreras, C. A., Garibay-Escobar, A., Canett-Romero, R., et al. (2013). Effects of dietary zinc manipulation on growth performance, zinc status and immune response during Giardia lamblia infection: a study in CD-1 mice. Nutrients 5, 3447-3460. doi: $10.3390 /$ nu5093447

Isaeva, G., and Efimova, N. G. (2010). Gastrointestinal giardiasis associated with Helicobacter pylori. Eksp. Klin. Gastroenterol. 30-34.

Ivanov, I., Atarashi, K., Manel, N., Brodie, E. L., Shima, T., Karaoz, U., et al. (2009). Induction of intestinal Th17 cells by segmented filamentous bacteria. Cell 139, 485-498. doi: 10.1016/j.cell.2009.09.033

Jakobsson, H. E., Rodriguez-Pineiro, A. M., Schutte, A., Ermund, A., Boysen, P., Bemark, M., et al. (2015). The composition of the gut microbiota shapes the colon mucus barrier. EMBO Rep. 16, 164-177. doi: 10.15252/embr.201439263

Jarroll, E. L., Keulen, H. V., Paget, T. A., and Lindmark, D. G. (2011). Giardia metabolism. Vienna: Springer.

Jarroll, E. L., Manning, P., Berrada, A., Hare, D., and Lindmark, D. G. (1989). Biochemistry and metabolism of Giardia. J. Protozool. 36, 190-197. doi: 10.1111/j.1550-7408.1989.tb01073.x

Jefferson, K. K. (2004). What drives bacteria to produce a biofilm? FEMS Microbiol. Lett. 236, 163-173. doi: 10.1016/j.femsle.2004.06.005
Jimenez, J. C., Fontaine, J., Creusy, C., Fleurisse, L., Grzych, J. M., Capron, M., et al. (2014). Antibody and cytokine responses to Giardia excretory/secretory proteins in Giardia intestinalis-infected BALB/c mice. Parasitol. Res. 113, 2709-2718. doi: 10.1007/s00436-014-3927-4

Johansson, M. E., Jakobsson, H. E., Holmen-Larsson, J., Schutte, A., Ermund, A., Rodriguez-Pineiro, A. M., et al. (2015). Normalization of host intestinal mucus layers requires long-term microbial colonization. Cell Host Microbe 18, 582-592. doi: 10.1016/j.chom.2015.10.007

Jung, Y., Wen, T., Mingler, M. K., Caldwell, J. M., Wang, Y. H., Chaplin, D. D., et al. (2015). IL-1beta in eosinophil-mediated small intestinal homeostasis and IgA production. Mucosal Immunol. 8, 930-942. doi: 10.1038/mi.2014.123

Kastl, A. J. Jr., Terry, N. A., Wu, G. D., and Albenberg, L. G. (2020). The structure and function of the human small intestinal microbiota: current understanding and future directions. Cell. Mol. Gastroenterol. Hepatol. 9, 33-45. doi: 10.1016/j.jcmgh.2019.07.006

Keselman, A., Li, E., Maloney, J., and Singer, S. M. (2016). The microbiota contributes to $\mathrm{CD}^{+} \mathrm{T}$ cell activation and nutrient malabsorption following intestinal infection with Giardia duodenalis. Infect. Immun. 84, 2853-2860. doi: 10.1128/IAI.00348-16

Khatami, S. S., Mukunda, B., and Ravakhah, K. (2004). Coinfection with Giardia lamblia and Clostridium difficile after use of ranitidine. Am. J. Med. Sci. 327, 91-93. doi: 10.1128/IAI.00348-16

Kim, J. H., Sohn, H. J., Yoo, J. K., Kang, H., Seong, G. S., Chwae, Y. J., et al. (2016). NLRP3 inflammasome activation in THP-1 target cells triggered by pathogenic Naegleria fowleri. Infect. Immun. 84, 2422-2428. doi: 10.1128/IAI.00275-16

Koopman, J. P., Stadhouders, A. M., Kennis, H. M., and De Boer, H. (1987). The attachment of filamentous segmented micro-organisms to the distal ileum wall of the mouse: a scanning and transmission electron microscopy study. Lab. Anim. 21, 48-52. doi: 10.1258/002367787780740743

Koru, O., Araz, E., Inci, A., and Tanyuksel, M. (2006). Co-infection of Giardia intestinalis and Cyclospora cayetanensis in an immunocompetent patient with prolonged diarrhea: case report. J. Microbiol. 44, 360-362.

Langford, T. D., Housley, M. P., Boes, M., Chen, J., Kagnoff, M. F., Gillin, F. D., et al. (2002). Central importance of immunoglobulin $\mathrm{A}$ in host defense against Giardia spp. Infect. Immun. 70, 11-18. doi: 10.1128/iai.70.1.11-18.2002

Leitch, G. J., Visvesvara, G. S., Wahlquist, S. P., and Harmon, C. T. (1989). Dietary fiber and giardiasis: dietary fiber reduces rate of intestinal infection by Giardia lamblia in the gerbil. Am. J. Trop. Med. Hyg. 41, 512-520. doi: 10.4269/ajtmh.1989.41.512

Lengerich, E. J., Addiss, D. G., and Juranek, D. D. (1994). Severe giardiasis in the United States. Clin. Infect. Dis. 18, 760-763. doi: 10.1093/clinids/18.5.760

Li, E., Zhou, P., Petrin, Z., and Singer, S. M. (2004). Mast cell-dependent control of Giardia lamblia infections in mice. Infect. Immun. 72, 6642-6649. doi: 10.1128/IAI.72.11.6642-6649.2004

Lima, A. A., Soares, A. M., Lima, N. L., Mota, R. M., Maciel, B. L., Kvalsund, M. P., et al. (2010). Effects of vitamin A supplementation on intestinal barrier function, growth, total parasitic, and specific Giardia spp infections in Brazilian children: a prospective randomized, double-blind, placebo-controlled trial. J. Pediatr. Gastroenterol. Nutr. 50, 309-315. doi: 10.1097/MPG.0b013e3181a96489

Lin, L., and Zhang, J. (2017). Role of intestinal microbiota and metabolites on gut homeostasis and human diseases. BMC Immunol. 18:2. doi: 10.1186/ s12865-016-0187-3

Lindmark, D. G., and Jarroll, E. L. (1982). Pyrimidine metabolism in Giardia lamblia trophozoites. Mol. Biochem. Parasitol. 5, 291-296. doi 10.1016/0166-6851(82)90036-6

Litvak, Y., Byndloss, M. X., Tsolis, R. M., and Baumler, A. J. (2017). Dysbiotic Proteobacteria expansion: a microbial signature of epithelial dysfunction. Curr. Opin. Microbiol. 39, 1-6. doi: 10.1016/j.mib.2017.07.003

Liu, J., Fu, Z., Hellman, L., and Svard, S. G. (2019). Cleavage specificity of recombinant Giardia intestinalis cysteine proteases: degradation of immunoglobulins and defensins. Mol. Biochem. Parasitol. 227, 29-38. doi: 10.1016/j.molbiopara.2018.10.004

Liu, J., Ma'ayeh, S., Peirasmaki, D., Lundstrom-Stadelmann, B., Hellman, L., and Svard, S. G. (2018). Secreted Giardia intestinalis cysteine proteases disrupt intestinal epithelial cell junctional complexes and degrade chemokines. Virulence 9, 879-894. doi: 10.1080/21505594.2018.1451284

Lozupone, C. A., Stombaugh, J. I., Gordon, J. I., Jansson, J. K., and Knight, R. (2012). Diversity, stability and resilience of the human gut microbiota. Nature 489, 220-230. doi: 10.1038/nature11550 
Lynch, S. V., and Pedersen, O. (2016). The human intestinal microbiome in health and disease. N. Engl. J. Med. 375, 2369-2379. doi: 10.1056/NEJMral 600266

Magne, D., Chochillon, C., Favennec, L., Kapel, N., Savel, J., and Gobert, J. G. (1994). Intestinal lipid metabolism in suckling rats infected with Giardia duodenalis. Parasitol. Res. 80, 528-530. doi: 10.1007/BF00932702

Manko-Prykhoda, A., Allain, T., Motta, J. P., Cotton, J. A., Feener, T., Oyeyemi, A., et al. (2020). Giardia spp. promote the production of antimicrobial peptides and attenuate disease severity induced by attaching and effacing enteropathogens via the induction of the NLRP3 inflammasome. Int. J. Parasitol. 50, 263-275. doi: 10.1016/j.ijpara.2019.12.011

Manko, A., Motta, J. P., Cotton, J. A., Feener, T., Oyeyemi, A., Vallance, B. A., et al. (2017). Giardia co-infection promotes the secretion of antimicrobial peptides beta-defensin 2 and trefoil factor 3 and attenuates attaching and effacing bacteria-induced intestinal disease. PLoS One 12:e178647. doi: 10.1371/journal.pone.0178647

Markowiak, P., and Slizewska, K. (2017). Effects of probiotics, prebiotics, and synbiotics on human health. Nutrients 9:1021. doi: 10.3390/nu9091021

Mckenney, E. A., Greene, L. K., Drea, C. M., and Yoder, A. D. (2017). Down for the count: Cryptosporidium infection depletes the gut microbiome in Coquerel's sifakas. Microb. Ecol. Health Dis. 28:1335165. doi: 10.1080/16512235. 2017.1335165

Mejia, R., Damania, A., Jeun, R., Bryan, P. E., Vargas, P., Juarez, M., et al. (2020). Impact of intestinal parasites on microbiota and cobalamin gene sequences: a pilot study. Parasit. Vectors 13:200. doi: 10.1186/s13071-02004073-7

Moreira, E. D. Jr., Nassri, V. B., Santos, R. S., Matos, J. F., De Carvalho, W. A., Silvani, C. S., et al. (2005). Association of Helicobacter pylori infection and giardiasis: results from a study of surrogate markers for fecal exposure among children. World J. Gastroenterol. 11, 2759-2763. doi: 10.3748/wjg. v11.i18.2759

Morken, M. H., Nysaeter, G., Strand, E. A., Hausken, T., and Berstad, A. (2008). Lactulose breath test results in patients with persistent abdominal symptoms following Giardia lamblia infection. Scand. J. Gastroenterol. 43, 141-145. doi: 10.1080/00365520701673960

Mortimer, L., Moreau, F., Cornick, S., and Chadee, K. (2014). Gal-lectin-dependent contact activates the inflammasome by invasive Entamoeba histolytica. Mucosal Immunol. 7, 829-841. doi: 10.1038/mi.2013.100

Mortimer, L., Moreau, F., Cornick, S., and Chadee, K. (2015). The NLRP3 inflammasome is a pathogen sensor for invasive Entamoeba histolytica via activation of alpha5betal integrin at the macrophage-amebae intercellular junction. PLoS Pathog. 11:e1004887. doi: 10.1371/journal.ppat.1004887

Moyano, S., Musso, J., Feliziani, C., Zamponi, N., Frontera, L. S., Ropolo, A. S., et al. (2019). Exosome biogenesis in the protozoa parasite Giardia lamblia: a model of reduced interorganellar crosstalk. Cell 8:1600. doi: 10.3390/ cells 8121600

Muhsen, K., Cohen, D., and Levine, M. M. (2014). Can Giardia lamblia infection lower the risk of acute diarrhea among preschool children? J. Trop. Pediatr. 60, 99-103. doi: 10.1093/tropej/fmt085

Mukherjee, A. K., Chowdhury, P., Rajendran, K., Nozaki, T., and Ganguly, S. (2014). Association between Giardia duodenalis and coinfection with other diarrhea-causing pathogens in India. Biomed. Res. Int. 2014:786480. doi: $10.1155 / 2014 / 786480$

Muller, N., and von Allmen, N. (2005). Recent insights into the mucosal reactions associated with Giardia lamblia infections. Int. J. Parasitol. 35, 1339-1347. doi: 10.1016/j.ijpara.2005.07.008

Nagao-Kitamoto, H., Kitamoto, S., Kuffa, P., and Kamada, N. (2016). Pathogenic role of the gut microbiota in gastrointestinal diseases. Intest. Res. 14, 127-138. doi: $10.5217 /$ ir.2016.14.2.127

Nieves-Ramirez, M. E., Partida-Rodriguez, O., Laforest-Lapointe, I., Reynolds, L. A., Brown, E. M., Valdez-Salazar, A., et al. (2018). Asymptomatic intestinal colonization with protist blastocystis is strongly associated with distinct microbiome ecological patterns. mSystems 3, e00007-e000018. doi: 10.1128/ mSystems.00007-18

Ortega-Pierres, G., Arguello-Garcia, R., Laredo-Cisneros, M. S., Fonseca-Linan, R., Gomez-Mondragon, M., Inzunza-Arroyo, R., et al. (2018). Giardipain-1, a protease secreted by Giardia duodenalis trophozoites, causes junctional, barrier and apoptotic damage in epithelial cell monolayers. Int. J. Parasitol. 48, 621-639. doi: 10.1016/j.ijpara.2018.01.006
Paerewijck, O., Maertens, B., Gagnaire, A., De Bosscher, K., and Geldhof, P. (2019). Delayed development of the protective IL-17A response following a Giardia muris infection in neonatal mice. Sci. Rep. 9:8959. doi: 10.1038/ s41598-019-45544-X

Perez, P. F., Minnaard, J., Rouvet, M., Knabenhans, C., Brassart, D., De Antoni, G. L., et al. (2001). Inhibition of Giardia intestinalis by extracellular factors from Lactobacilli: an in vitro study. Appl. Environ. Microbiol. 67, 5037-5042. doi: 10.1128/AEM.67.11.5037-5042.2001

Perrucci, S., Fichi, G., Ricci, E., Galosi, L., Lalle, M., and Rossi, G. (2019). In vitro and ex vivo evaluation of the anti-Giardia duodenalis activity of the supernatant of Slab51 (SivoMixx). PLoS One 14:e0213385. doi: 10.1371/ journal.pone.0213385

Pique, N., Berlanga, M., and Minana-Galbis, D. (2019). Health benefits of heat-killed (Tyndallized) probiotics: an overview. Int. J. Mol. Sci. 20:2534. doi: 10.3390/ijms20102534

Platts-Mills, J. A., Babji, S., Bodhidatta, L., Gratz, J., Haque, R., Havt, A., et al. (2015). Pathogen-specific burdens of community diarrhoea in developing countries: a multisite birth cohort study (MAL-ED). Lancet Glob. Health 3, e564-e575. doi: 10.1016/S2214-109X(15)00151-5

Quihui-Cota, L., Mendez Estrada, R. O., Astiazaran-Garcia, H., Morales-Figueroa, G. G., Moreno-Reyes, M. J., Cuadras-Romo, D., et al. (2012). Changes in serum zinc levels associated with giardiasis and dietary zinc intake in mice. Biol. Trace Elem. Res. 145, 396-402. doi: 10.1007/s12011-011-9208-5

Ras, R., Huynh, K., Desoky, E., Badawy, A., and Widmer, G. (2015). Perturbation of the intestinal microbiota of mice infected with Cryptosporidium parvum. Int. J. Parasitol. 45, 567-573. doi: 10.1016/j.ijpara.2015.03.005

Riba, A., Hassani, K., Walker, A., Van Best, N., Von Zeschwitz, D., Anslinger, T., et al. (2020). Disturbed gut microbiota and bile homeostasis in Giardiainfected mice contributes to metabolic dysregulation and growth impairment. Sci. Transl. Med. 12:eaay7019. doi: 10.1126/scitranslmed.aay7019

Ribeiro, M. R. S., Oliveira, D. R., Oliveira, F. M. S., Caliari, M. V., Martins, F. S., Nicoli, J. R., et al. (2018). Effect of probiotic Saccharomyces boulardii in experimental giardiasis. Benef. Microbes 9, 789-797. doi: 10.3920/BM2017.0155

Rinninella, E., Raoul, P., Cintoni, M., Franceschi, F., Miggiano, G. A. D., Gasbarrini, A., and Mele, M. C. (2019). What is the healthy gut microbiota composition? A changing ecosystem across age, environment, diet, and diseases. Microorganisms 7:14. doi: 10.3390/microorganisms7010014

Rodrigues, M. A., De-Carvalho, M. F., and Montenegro, M. R. (1994). Effects of protein deficiency and natural intestinal infection with Giardia lamblia on jejunal intraepithelial lymphocytes in rats of different ages. Braz. J. Med. Biol. Res. 27, 1347-1353.

Ryan, U., and Caccio, S. M. (2013). Zoonotic potential of Giardia. Int. J. Parasitol. 43, 943-956. doi: 10.1016/j.ijpara.2013.06.001

Scanlan, P. D., Stensvold, C. R., Rajilic-Stojanovic, M., Heilig, H. G., De Vos, W. M., O'toole, P. W., et al. (2014). The microbial eukaryote Blastocystis is a prevalent and diverse member of the healthy human gut microbiota. FEMS Microbiol. Ecol. 90, 326-330. doi: 10.1111/1574-6941.12396

Schroeder, B. O. (2019). Fight them or feed them: how the intestinal mucus layer manages the gut microbiota. Gastroenterol. Rep. 7, 3-12. doi: 10.1093/ gastro/goy052

Scott, K. G., Yu, L. C., and Buret, A. G. (2004). Role of $\mathrm{CD}^{+}$and CD4 ${ }^{+} \mathrm{T}$ lymphocytes in jejunal mucosal injury during murine giardiasis. Infect. Immun. 72, 3536-3542. doi: 10.1128/IAI.72.6.3536-3542.2004

Shea-Donohue, T., Qin, B., and Smith, A. (2017). Parasites, nutrition, immune responses and biology of metabolic tissues. Parasite Immunol. 39:10.1111/ pim.12422. doi: 10.1111/pim.12422

Shreiner, A. B., Kao, J. Y., and Young, V. B. (2015). The gut microbiome in health and in disease. Curr. Opin. Gastroenterol. 31, 69-75. doi: 10.1097/ MOG.0000000000000139

Shukla, G., Bhatia, R., and Sharma, A. (2016). Prebiotic inulin supplementation modulates the immune response and restores gut morphology in Giardia duodenalis-infected malnourished mice. Parasitol. Res. 115, 4189-4198. doi: 10.1007/s00436-016-5196-x

Shukla, G., Devi, P., and Sehgal, R. (2008). Effect of Lactobacillus casei as a probiotic on modulation of giardiasis. Dig. Dis. Sci. 53, 2671-2679. doi: 10.1007/s10620-007-0197-3

Shukla, G., and Sidhu, R. K. (2011). Lactobacillus casei as a probiotic in malnourished Giardia lamblia-infected mice: a biochemical and histopathological study. Can. J. Microbiol. 57, 127-135. doi: 10.1139/w10-110 
Shukla, G., Singh, S., and Verma, A. (2013). Oral administration of the probiotic Lactobacillus casei ameliorates gut morphology and physiology in malnourishedGiardia intestinalis-infected BALB/c mice. ISRN Parasitol. 2013:762638. doi: $10.5402 / 2013 / 762638$

Simsek, I. (2011). Irritable bowel syndrome and other functional gastrointestinal disorders. J. Clin. Gastroenterol. 45, S86-S88. doi: 10.1097/MCG.0b013e3 $1821 \mathrm{fbd} 6 \mathrm{f}$

Singer, S. M., Fink, M. Y., and Angelova, V. V. (2019). Recent insights into innate and adaptive immune responses to Giardia. Adv. Parasitol. 106, 171-208. doi: 10.1016/bs.apar.2019.07.004

Singer, S. M., and Nash, T. E. (2000). The role of normal flora in Giardia lamblia infections in mice. J. Infect. Dis. 181, 1510-1512. doi: 10.1086/315409

Slapeta, J., Dowd, S. E., Alanazi, A. D., Westman, M. E., and Brown, G. K. (2015). Differences in the faecal microbiome of non-diarrhoeic clinically healthy dogs and cats associated with Giardia duodenalis infection: impact of hookworms and coccidia. Int. J. Parasitol. 45, 585-594. doi: 10.1016/j. ijpara.2015.04.001

Sorokulova, I. (2008). Preclinical testing in the development of probiotics: a regulatory perspective with Bacillus strains as an example. Clin. Infect. Dis. 46(Suppl. 2), S92-S95. doi: 10.1086/523334

Stadelmann, B., Hanevik, K., Andersson, M. K., Bruserud, O., and Svard, S. G. (2013). The role of arginine and arginine-metabolizing enzymes during Giardia-host cell interactions in vitro. BMC Microbiol. 13:256. doi: 10.1186/1471-2180-13-256

Stadelmann, B., Merino, M. C., Persson, L., and Svärd, S. G. (2012). Arginine consumption by the intestinal parasite Giardia intestinalis reduces proliferation of intestinal epithelial cells. PLoS One 7:e45325. doi: 10.1371/journal. pone. 0045325

Stafford, K., Kollasch, T. M., Duncan, K. T., Horr, S., Goddu, T., Heinz-Loomer, C., et al. (2020). Detection of gastrointestinal parasitism at recreational canine sites in the USA: the DOGPARCS study. Parasit. Vectors 13:275. doi: 10.1186/ s13071-020-04147-6

Suh, M., Belosevic, M., and Clandinin, M. T. (2004). Dietary lipids containing gangliosides reduce Giardia muris infection in vivo and survival of Giardia lamblia trophozoites in vitro. Parasitology 128, 595-602. doi: 10.1017/ s0031182004005128

Tako, E. A., Hassimi, M. F., Li, E., and Singer, S. M. (2013). Transcriptomic analysis of the host response to Giardia duodenalis infection reveals redundant mechanisms for parasite control. MBio 4, e00660-e006613. doi: 10.1128/ mBio.00660-13

Tandon, B. N., Tandon, R. K., Satpathy, B. K., and Shriniwas, (1977). Mechanism of malabsorption in giardiasis: a study of bacterial flora and bile salt deconjugation in upper jejunum. Gut 18, 176-181. doi: 10.1136/gut.18.3.176

Taverniti, V., and Guglielmetti, S. (2011). The immunomodulatory properties of probiotic microorganisms beyond their viability (ghost probiotics: proposal of paraprobiotic concept). Genes Nutr. 6, 261-274. doi: 10.1007/ s12263-011-0218-x

Tomkins, A. M., Wright, S. G., Drasar, B. S., and James, W. P. (1978). Bacterial colonization of jejunal mucosa in giardiasis. Trans. R. Soc. Trop. Med. Hyg. 72, 33-36. doi: 10.1016/0035-9203(78)90294-8

Toro-Londono, M. A., Bedoya-Urrego, K., Garcia-Montoya, G. M., Galvan-Diaz, A. L., and Alzate, J. F. (2019). Intestinal parasitic infection alters bacterial gut microbiota in children. PeerJ 7:e6200. doi: 10.7717/ peerj. 6200

Torres, M. F., Uetanabaro, A. P. T., Costa, A. F., Alves, C. A., Farias, L. M., Bambirra, E. A., et al. (2000). Influence of bacteria from the duodenal microbiota of patients with symptomatic giardiasis on the pathogenicity of Giardia duodenalis in gnotoxenic mice. J. Med. Microbiol. 49, 209-215. doi: 10.1099/0022-1317-49-3-209

Torres, M. R., Silva, M. E., Vieira, E. C., Bambirra, E. A., Sogayar, M. I., Pena, F. J., et al. (1992). Intragastric infection of conventional and germfree mice with Giardia lamblia. Braz. J. Med. Biol. Res. 25, 349-352.

Travers, M. A., Florent, I., Kohl, L., and Grellier, P. (2011). Probiotics for the control of parasites: an overview. J. Parasitol. Res. 2011:610769. doi: $10.1155 / 2011 / 610769$

Travers, M. A., Sow, C., Zirah, S., Deregnaucourt, C., Chaouch, S., Queiroz, R. M., et al. (2016). Deconjugated bile salts produced by extracellular bile-salt hydrolase-like activities from the probiotic Lactobacillus johnsonii La1 inhibit
Giardia duodenalis in vitro growth. Front. Microbiol. 7:1453. doi: 10.3389/ fmicb.2016.01453

Tropini, C., Earle, K. A., Huang, K. C., and Sonnenburg, J. L. (2017). The gut microbiome: connecting spatial organization to function. Cell Host Microbe 21, 433-442. doi: 10.1016/j.chom.2017.03.010

Trujillo-de Santiago, G., Lobo-Zegers, M. J., Montes-Fonseca, S. L., Zhang, Y. S., and Alvarez, M. M. (2018). Gut-microbiota-on-a-chip: an enabling field for physiological research. Microphysiol. Syst. 2, 1-23. doi: 10.21037/mps.2018.09.01

Tuddenham, S., and Sears, C. L. (2015). The intestinal microbiome and health. Curr. Opin. Infect. Dis. 28, 464-470. doi: 10.1097/QCO.0000000000000196

Tysnes, K. R., Skancke, E., and Robertson, L. J. (2014). Subclinical Giardia in dogs: a veterinary conundrum relevant to human infection. Trends Parasitol. 30, 520-527. doi: 10.1016/j.pt.2014.08.007

Upadhyay, P., Ganguly, N. K., Mahajan, R. C., and Walia, B. N. (1985). Intestinal uptake of nutrients in normal and malnourished animals infected with Giardia lamblia. Digestion 32, 243-248. doi: 10.1159/000199244

Veenemans, J., Mank, T., Ottenhof, M., Baidjoe, A., Mbugi, E. V., Demir, A. Y., et al. (2011). Protection against diarrhea associated with Giardia intestinalis is lost with multi-nutrient supplementation: a study in Tanzanian children. PLoS Negl. Trop. Dis. 5:e1158. doi: 10.1371/journal.pntd.0001158

Ventura, L. L., Oliveira, D. R., Viana, J. C., Santos, J. F., Caliari, M. V., and Gomes, M. A. (2013). Impact of protein malnutrition on histological parameters of experimentally infected animals with Giardia lamblia. Exp. Parasitol. 133, 391-395. doi: 10.1016/j.exppara.2013.01.007

Verma, A. K., Verma, R., Ahuja, V., and Paul, J. (2012). Real-time analysis of gut flora in Entamoeba histolytica infected patients of northern India. BMC Microbiol. 12:183. doi: 10.1186/1471-2180-12-183

Wang, J., Kurilshikov, A., Radjabzadeh, D., Turpin, W., Croitoru, K., Bonder, M. J., et al. (2018). Meta-analysis of human genome-microbiome association studies: the MiBioGen consortium initiative. Microbiome 6:101. doi: 10.1186/ s40168-018-0479-3

Wang, L., Xiao, L., Duan, L., Ye, J., Guo, Y., Guo, M., et al. (2013). Concurrent infections of Giardia duodenalis, Enterocytozoon bieneusi, and Clostridium difficile in children during a cryptosporidiosis outbreak in a pediatric hospital in China. PLoS Negl. Trop. Dis. 7:e2437. doi: 10.1371/journal.pntd.0002437

Wang, M., Ahrne, S., Jeppsson, B., and Molin, G. (2005). Comparison of bacterial diversity along the human intestinal tract by direct cloning and sequencing of 16S rRNA genes. FEMS Microbiol. Ecol. 54, 219-231. doi: 10.1016/j.femsec.2005.03.012

$\mathrm{Wu}, \mathrm{H}$. J., and Wu, E. (2012). The role of gut microbiota in immune homeostasis and autoimmunity. Gut Microbes 3, 4-14. doi: 10.4161/gmic.19320

Yordanova, I. A., Cortes, A., Klotz, C., Kuhl, A. A., Heimesaat, M. M., Cantacessi, C., et al. (2019). RORgammat(+) Treg to Th17 ratios correlate with susceptibility to Giardia infection. Sci. Rep. 9:20328. doi: 10.1038/ s41598-019-56416-9

Zeyrek, D., Zeyrek, F., Cakmak, A., and Cekin, A. (2008). Association of Helicobacter pylori and giardiasis in children with recurrent abdominal pain. Turkiye Parazitol. Derg. 32, 4-7.

Zhou, P., Li, E., Zhu, N., Robertson, J., Nash, T., and Singer, S. M. (2003). Role of interleukin-6 in the control of acute and chronic Giardia lamblia infections in mice. Infect. Immun. 71, 1566-1568. doi: 10.1128/ iai.71.3.1566-1568.2003

Zhou, Q., Zhang, B., and Verne, G. N. (2009). Intestinal membrane permeability and hypersensitivity in the irritable bowel syndrome. Pain 146, 41-46. doi 10.1016/j.pain.2009.06.017

Conflict of Interest: The authors declare that the research was conducted in the absence of any commercial or financial relationships that could be construed as a potential conflict of interest.

The handling editor declared a past co-authorship with one of the authors $\mathrm{AB}$.

Copyright (C) 2021 Fekete, Allain, Siddiq, Sosnowski and Buret. This is an open-access article distributed under the terms of the Creative Commons Attribution License (CC BY). The use, distribution or reproduction in other forums is permitted, provided the original author(s) and the copyright owner(s) are credited and that the original publication in this journal is cited, in accordance with accepted academic practice. No use, distribution or reproduction is permitted which does not comply with these terms. 